\title{
A strong averaging principle for Lévy diffusions in foliated spaces with unbounded leaves.
}

\author{
Paulo Henrique da Costa $* \quad$ Michael A. Högele ${ }^{\dagger} \quad$ Paulo Regis Ruffino ${ }^{\ddagger}$
}

July 15, 2021

\begin{abstract}
This article extends a strong averaging principle for Lévy diffusions which live on the leaves of a foliated manifold subject to small transversal Lévy type perturbation to the case of noncompact leaves. The main result states that the existence of $p$-th moments of the foliated Lévy diffusion for $p \geqslant 2$ and an ergodic convergence of its coefficients in $L^{p}$ implies the strong $L^{p}$ convergence of the fast perturbed motion on the time scale $t / \varepsilon$ to the system driven by the averaged coefficients. In order to compensate the non-compactness of the leaves we use an estimate of the dynamical system for each of the increments of the canonical Marcus equation derived in [7, the boundedness of the coefficients in $L^{p}$ and a nonlinear Gronwall-Bihari type estimate. The price for the non-compactness are slower rates of convergence, given as $p$-dependent powers of $\varepsilon$ strictly smaller than $1 / 4$.
\end{abstract}

Keywords: strong averaging principle; scale separation; averaging of slow-fast diffusions; Lévy jump diffusions on manifolds; foliated manifolds; Marcus canonical equation; 2010 Mathematical Subject Classification: 60H10, 60J75, 60F15, 60G51, 58J65, 58J37, 70K65, 37H10.

\section{Introduction}

The literature on averaging principles for deterministic and stochastic systems reaches far back to the 18th century and is enormously rich both in theory and applications. At this point, however, we would like to refrain from a more systematic review of the long and bifurcated history of the field and restrict ourselves to the references to some classical texts. Standard texts on the deterministic field include [3], 28], 30], 31] and the references therein. For stochastic systems we refer to [9], [13], [15, [16], 27], 22], 6] and [4] and the respective bibliographies.

Loosely speaking, an averaging principle describes the observation that in a coupled slow-fast system in the limit of infinite time scale separation, the slow system is close to a system, where the fast variable is replaced by the limiting measure of its ergodic time average. In the case of stochastic differential equations rescaling time show that this problem can be restated as a problem of an ergodic system perturbed by small perturbations.

The results of this article generalize recent approaches by the authors for diffusions on finite dimensional foliated manifolds. For properties of foliated spaces consult [5], [11, [29], [32]. Motivated by [21] Gargate and Ruffino studied in [10] the case of foliated Gaussian diffusions on compact

\footnotetext{
*Departamento de Matemática, Universidade de Brasília, Brasília, Brazil; phcosta@unb.br

${ }^{\dagger}$ Departamento de Matemáticas, Universidad de los Andes, Bogotá, Colombia; ma.hoegele@uniandes.edu.co

${ }^{\ddagger}$ IMECC, Universidade Estadual de Campinas, Campinas, Brazil; ruffino@ime.unicamp.br
} 
leaves subject to deterministic Lipschitz transversal perturbation. In Högele and Ruffino [12] the authors treat the case of foliated Lévy jump diffusions with exponential moments but still with deterministic transversal perturbation and compact leaves. This type of processes is described in terms of canonical Marcus equations.

The recent work by da Costa and Högele [7] covers the case of a general class of foliated Lévy diffusions on compact leaves perturbed by a near optimally large class of Lévy diffusions. This is carried out with the help of a nonlinear comparison principle and a fine study of the individual jump increments. However in that case the compactness still allows global estimates of the horizontal components, for instance, in the force acting on the "vertical" component of the perturbed system.

This article treats an averaging principle for the same type of foliated Lévy diffusions, however with non-compact leaves. The lack of compactness yields an almost unmitigated system of fully coupled SDEs. The strategies are once again non-linear Gronwall-Bihari type inequalities, using the $L^{p}$ boundedness of the drift. However, this comes at the price of slower rates of convergence. Our main result, Theorem 2.4 states that locally the transversal behavior of $X_{\frac{t}{\varepsilon}}^{\varepsilon}$ can be approximated $L^{p}$ uniformly in time by the Lévy stochastic differential equation in the transversal space with coefficients given by the average of the deterministic transversal component of the perturbation (with respect to the invariant measure on the leaves for the original unperturbed dynamics) and the diffusion component given by the projection of the original perturbation into the transversal space. We should mention that our results cover the results by [8] as the special case of uniformly bounded jumps.

In the Section 2 we present the dynamical and stochastic framework, the main hypotheses and the main result. In Section 3 we prove the key proposition which is the basis for the proof of the main theorem, proved in Section 4. Wherever possible in the exposition without lost of coherence we refer to the article [7] in order to avoid trivial repetition.

\section{Object of study and main results}

\subsection{The setup}

The following setup is a non-compact extension of the setup on [7] and [12].

The foliated manifold: Let $M$ be a finite dimensional connected, smooth Riemannian manifold. It is known by the classical Nash theorem in 23 , that any finite dimensional smooth manifold may be embedded in $\mathbb{R}^{m}$ with $m$ sufficiently large. We assume that $M$ is equipped with an $n$ dimensional foliation $\mathfrak{M}$ in the following sense. Let $\mathfrak{M}=\left(L_{x}\right)_{x \in M}$, with $M=\bigcup_{x \in M} L_{x}$ and the sets $L_{x}$ are equivalence classes of the elements of $M$ satisfying the following.

a. Given $x_{0} \in M$ there exist a neighborhood $U \subset M$ of the corresponding leaf $L_{x_{0}}$ and a diffeomorphism $\varphi: U \rightarrow L_{x_{0}} \times V$, where $V \subset \mathbb{R}^{d}$ is a connected open set containing the origin $0 \in \mathbb{R}^{d}$.

b. For any $L_{x_{0}} \in \mathfrak{M}$ the neighborhood $U \supset L_{x_{0}}$ can be taken small enough such that the coordinate map $\varphi$ is uniformly Lipschitz continuous.

Remark 2.1 The second coordinate of a point $x \in U$, called the vertical coordinate, will be denoted with the help the projection $\pi: U \rightarrow V$ by $\varphi(x)=(\bar{x}, \pi(x))$ for some $\bar{x} \in L_{x}$. For any fixed $v \in V$, the preimage $\pi^{-1}(v)$ is the leaf $L_{x}$, where $x$ is any point in $U$ such that the vertical projection satisfies $\pi(x)=v$. 
The unperturbed equation: We are interested in the ergodic behavior of the strong solution of a Lévy driven SDE with jump components which takes values in $M$ and which respects the foliation. Intuitively, a straight line increment $z$ does not cause the exit from the leaf of its current position if the entire line segment $\left(x_{0}+\theta z\right)_{\theta \in[0,1]}$ is contained in it. Ordinary differential equations with a vector field $F$ on the right-hand side generalize this concept in the following sense. By definition, their solutions follow $F$ as "infinitesimal" tangents. If $F$ itself is tangential to a given manifold the integral curves remain "infinitesimally tangential" to the manifold and hence will not leave it. Therefore a straight line jump increment $z$ which is transformed in the stochastic integral into an integral curve following a tangential vector field $F$ of a given leaf will remain on the leaf, that is, respect the foliated structure of the space. This intuition is made rigorous in the notion of stochastic integration in the sense of a canonical Marcus equation in the sense of Kurtz, Pardoux and Protter [18. Those equations are the equivalent for Lévy jump diffusions to the Stratonovich equation for Brownian SDE in that they satisfy the Leibniz chain rule (cf. Proposition 4.2 in [18]). Their definition however is different since they treat discontinuous processes.

Let us consider the formal canonical Marcus stochastic differential equation

$$
d X_{t}=F_{0}\left(X_{t}\right) d t+F\left(X_{t}\right) \diamond d Z_{t}+G\left(X_{t}\right) \circ d B_{t}, \quad X_{0}=x_{0} \in M,
$$

with the following components defined over a given filtered probability space $\boldsymbol{\Omega}=\left(\Omega, \mathcal{F},\left(\mathcal{F}_{t}\right)_{t \geqslant 0}, \mathbb{P}\right)$ which satisfies the usual conditions in the sense of Protter [25].

1. Let $Z=\left(Z_{t}\right)_{t \geqslant 0}$ with $Z_{t}=\left(Z_{t}^{1}, \ldots, Z_{t}^{r}\right)$ be a Lévy process over $\Omega$ with values in $\mathbb{R}^{r}$ for some $r \in \mathbb{N}$ and characteristic triplet $(0, \nu, 0)$. It is a consequence of the Lévy-Itô decomposition of $Z$ that $Z$ is a pure jump process with respect to a Lévy measure $\nu: \mathcal{B}\left(\mathbb{R}^{r}\right) \rightarrow[0, \infty]$ satisfying

$$
\int_{\mathbb{R}^{r}}\left(1 \wedge\|z\|^{2}\right) \nu(d z)<\infty \quad \text { and } \quad \nu(\{0\})=0 .
$$

For details we refer to the overview article by Kunita [20] and the monographs of Sato [26] or Applebaum [2].

2. Let $F \in \mathcal{C}^{2}\left(M ; L\left(\mathbb{R}^{r} ; T \mathfrak{M}\right)\right)$ satisfying the following. The function $x \mapsto F(x)$ is $\mathcal{C}^{2}$ and for each $x \in M$ the linear map $F(x)$ maps a vector $z \in \mathbb{R}^{r} \mapsto F(x) z \in T_{x} L_{x}$ to the tangent space of the respective leaf. Furthermore, let $F$ and $(D F) F$ be globally Lipschitz continuous on $M$ with common Lipschitz constant $\ell>0$.

3. Let $B=\left(B^{1}, \ldots, B^{r}\right)$ be an $\mathbb{R}^{r}$-valued Brownian motion on $\Omega$ and $G \in \mathcal{C}^{2}\left(M, L\left(\mathbb{R}^{r}, T \mathfrak{M}\right)\right)$. We assume that $G$ and $(D G) G$ are globally Lipschitz continuous on $M$ with Lipschitz constant $\ell>0$.

Following [18] a strong solution of the formal equation (11) is defined as a random map $X$ : $[0, \infty) \times \Omega \rightarrow M$ satisfying almost surely for all $t \geqslant 0$

$$
\begin{aligned}
X_{t}= & x_{0}+\int_{0}^{t} F_{0}\left(X_{s}\right) d s+\int_{0}^{t} G\left(X_{s}\right) d B_{s}+\frac{1}{2} \int_{0}^{t}\left(D G\left(X_{s}\right)\right) G\left(X_{s}\right) d\langle B\rangle_{s} \\
& +\int_{0}^{t} F\left(X_{s-}\right) d Z_{s}+\sum_{0<s \leqslant t}\left(\Phi^{F \Delta_{s} Z}\left(X_{s-}\right)-X_{s-}-F\left(X_{s-}\right) \Delta_{s} Z\right),
\end{aligned}
$$

where $\langle B\rangle$. stands for the quadratic variation process of $B$ in $\mathbb{R}^{r}$ and the function $\Phi^{F z}(x)=$ $Y(1, x ; F z)$ and $Y(t, x ; F z)$ for the solution of the ordinary differential equation

$$
\frac{d}{d \sigma} Y(\sigma)=F(Y(\sigma)) z, \quad Y(0)=x \in M, \quad z \in \mathbb{R}^{r}
$$


The perturbed equation: This article studies the situation where an SDE in the sense of (3), which is invariant on the leaf of the initial condition $x_{0}$ is perturbed by a transversal smooth vector field $\varepsilon K d t$ and stochastic differentials $\varepsilon \tilde{G} \circ d \tilde{B}$ and $\varepsilon \tilde{K} \diamond d \tilde{Z}, \varepsilon>0$, in the limit for $\varepsilon \searrow 0$. More precisely we denote by $X^{\varepsilon}, \varepsilon>0$ the analogous solution in the sense of (3) of the perturbed formal system

$$
\begin{aligned}
d X_{t}^{\varepsilon}= & F_{0}\left(X_{t}^{\varepsilon}\right) d t+F\left(X_{t}^{\varepsilon}\right) \diamond d Z_{t}+G\left(X_{t}^{\varepsilon}\right) \circ d B_{t} \\
& +\varepsilon\left(K\left(X_{t}^{\varepsilon}\right) d t+\tilde{K}\left(\pi\left(X_{t}^{\varepsilon}\right)\right) \diamond d \tilde{Z}_{t}+\tilde{G}\left(\pi\left(X_{t}^{\varepsilon}\right)\right) \circ d \tilde{B}_{t}\right), \\
X_{0}^{\varepsilon}= & x_{0} \in M
\end{aligned}
$$

where the additional coefficients are defined as follows.

4. The vector field $K: M \rightarrow T M$ is smooth and globally Lipschitz continuous.

5. Let $\tilde{Z}=\left(\tilde{Z}^{1}, \ldots, \tilde{Z}^{r}\right)$ be a Lévy process on $\boldsymbol{\Omega}$ with values in $\mathbb{R}^{r}$ with Lévy triple $\left(0, \nu^{\prime}, 0\right)$ for $\nu^{\prime}$ being a given Lévy measure. The vector field $\tilde{K} \in \mathcal{C}^{2}\left(V, L\left(\mathbb{R}^{r}, T M\right)\right)$ satisfies that $\tilde{K}$ and $(D \tilde{K}) \tilde{K}$ are globally Lipschitz continuous with Lipschitz constant $\tilde{\ell}>0$.

6. Let $\tilde{B}=\left(\tilde{B}^{1}, \ldots, \tilde{B}^{r}\right)$ be a $\mathbb{R}^{r}$-valued Brownian motion over $\Omega$ and $\tilde{G} \in \mathcal{C}^{2}\left(V, L\left(\mathbb{R}^{r}, T M\right)\right)$ satisfy that $\tilde{G}$ and $(D \tilde{G}) \tilde{G}$ are globally Lipschitz continous with Lipschitz constant $\tilde{\ell}>0$.

7. Assume that the stochastic processes $Z, B, \tilde{Z}, \tilde{B}$ are independent on $\boldsymbol{\Omega}$.

Theorem 2.2 ([18], Theorem 3.2 and 5.1) 1. Under the preceding setup (items a., b., 1.- 3. and \%.) there is a unique $\left(\mathcal{F}_{t}\right)_{t \geqslant 0}$ semimartingale $X$ which is a strong global solution of (1) in the sense of equation (3). It has a càdlàg version and is a (strong) Markov process.

2. Under the preceding setup (in particular items a., b. and 1.-7.) there is a unique semimartingale $X^{\varepsilon}$ which is a strong global solution of equation (5) in the sense of equation (3), where $F_{0}$ is replaced by $F_{0}+\varepsilon K$ and $F$ by $(F, \varepsilon \tilde{K}), G$ by $(G, \varepsilon \tilde{G}), B$ by $(B, \tilde{B})$ and $Z$ by $(Z, \tilde{Z})$. The perturbed solution $X^{\varepsilon}$ has càdlàg paths almost surely and is a (strong) Markov process.

The support theorem: We are now in the position to apply the crucial support theorem, Proposition 4.3, in Kurtz, Pardoux and Protter [18]. Under the hypotheses of Theorem 2.2 we have for any $\varepsilon>0$ that $x_{0} \in M$ implies that $\mathbb{P}\left(X_{t}^{\varepsilon}\left(x_{0}\right) \in M \forall t \geqslant 0\right)=1$. This result applied to the leaves of $\mathfrak{M}$ yields that each solution $X$ of (1) is foliated in the sense that $X$ stays on the leaf of its initial condition, i.e. for any $x_{0} \in M$ we have $\mathbb{P}\left(X_{t}\left(x_{0}\right) \in L_{x_{0}} \forall t \geqslant 0\right)=1$.

\subsection{The hypotheses and the main result}

In the general setup of Subsection 2.1 we assume the following precise hypotheses.

Hypothesis 1: Integrability. There is an exponent $p \geqslant 2$ such that the Lévy measures $\nu$ of $Z$ and $\nu^{\prime}$ of $\tilde{Z}$ satisfy

$$
\int_{\mathbb{R}^{r}}\|z\|^{p} \nu(d z)<\infty \quad \text { and } \quad \int_{\mathbb{R}^{r}}\|z\|^{2 p} \nu^{\prime}(d z)<\infty .
$$




\section{Hypothesis 2: Foliated invariant measures.}

1. Each leaf $L_{x_{0}} \in \mathfrak{M}$ passing through $x_{0} \in M$ has an associated unique invariant measure $\mu_{x_{0}}$ with $\operatorname{supp}\left(\mu_{x_{0}}\right)=L_{x_{0}}$ of the unperturbed foliated system (1) with initial condition $x_{0}$.

2. For $v_{0}=\pi\left(x_{0}\right)$ the vertical coordinate of $x_{0} \in M$ we define for $h: M \rightarrow T M$

$$
Q^{h}\left(v_{0}\right):=\int_{L_{x_{0}}} h(y) \mu_{x_{0}}(d y) .
$$

We assume for any globally Lipschitz continuous map $h: M \rightarrow T M$ the function

$$
\mathbb{R}^{d} \supset V \ni v \mapsto Q^{h}(v) \in \mathbb{R}^{d}
$$

is globally Lipschitz continuous.

Remark 2.3 Note that $L_{x_{0}}$ only depends on $v_{0}=\pi\left(x_{0}\right)$. The same is true for $\mu_{x_{0}}$.

Hypothesis 2 guarantees that for each $x_{0} \in M, v_{0}=\pi\left(x_{0}\right) \in V$ the stochastic differential equation

$$
d w_{t}=Q^{\pi K}\left(w_{t}\right) d t+\tilde{K}\left(w_{t}\right) \diamond d \tilde{Z}_{t}+\tilde{G}\left(w_{t}\right) \circ d \tilde{B}_{t}, \quad w_{0}=v_{0} \in V
$$

has a unique strong solution $w=\left(w_{t}\left(v_{0}\right)\right)_{t \in[0, \sigma)}$ on $\boldsymbol{\Omega}, \sigma$ being the first exit time of $w$ from $V$.

Hypothesis 3: Ergodic convergence of the vertical coefficient in $L^{p}$. Fix $p \geqslant 2$ from Hypothesis 1.

1. There are continuous functions $\eta^{0}:[0, \infty) \rightarrow[0, \infty)$ and $\bar{\eta}: M \rightarrow[0, \infty)$, where $\eta^{0}$ is monotonically decreasing with $\eta^{0}(t) \rightarrow 0$ as $t \rightarrow \infty$ and $\bar{\eta}$ is globally Lipschitz continuous. For all $x_{0} \in M$ and $t \geqslant 0$ we have

$$
\left(\mathbb{E}\left|\frac{1}{t} \int_{0}^{t} \pi K\left(X_{s}\left(x_{0}\right)\right) d s-Q^{\pi K}\left(\pi\left(x_{0}\right)\right)\right|^{p}\right)^{\frac{1}{p}} \leqslant \bar{\eta}\left(x_{0}\right) \eta^{0}(t) .
$$

2. We assume for any $x_{0} \in M$ that $\int \bar{\eta}(y) \mu_{x_{0}}(d y)<\infty$.

It is known in the literature that there is no standard rate of convergence [14], [17, which is why we assume an external rate of convergence, which decomposes by factors, see for instance [19].

For $\varepsilon>0$ and $x_{0} \in M$ let $\tau^{\varepsilon}$ being the first exit time of the solution $X^{\varepsilon}\left(x_{0}\right)$ of equation (5) from the foliated coordinate neighborhood $U$ of item a) in Subsection 2.1.

The main result of this article is the following strong averaging principle.

Theorem 2.4 Let Hypotheses 1, 2 and 3 be satisfied for some $p \geqslant 2$. Then for any $x_{0} \in M$ and $\lambda \in\left(0, \frac{p-1}{p^{2}}\right)$ there are constants $c, C>0$ and $\varepsilon_{0} \in(0,1]$ such that $\varepsilon \in\left(0, \varepsilon_{0}\right]$ and $T \in[0,1]$ imply

$$
\left(\mathbb{E}\left[\sup _{t \in\left[0, T \wedge \varepsilon \tau^{\varepsilon} \wedge \sigma\right]}\left|\pi\left(X_{\frac{t}{\varepsilon}}^{\varepsilon}\left(x_{0}\right)\right)-w_{t}\left(\pi\left(x_{0}\right)\right)\right|^{p}\right]\right)^{\frac{1}{p}} \leqslant C T\left[\varepsilon^{\lambda}+\eta^{0}(c T|\ln (\varepsilon)|)\right] .
$$

Remark 2.5 Our results focus on the case with only $p$-th moments, hence we set the coefficients $G$ and $\tilde{G}$ to zero in the proofs. 


\section{The transversal perturbations}

In order to prove the main theorem we need to control the error $X^{\varepsilon}-X$ in terms of $L^{p}$. This section is dedicated to the control of this error by the following result.

Proposition 3.1 Let the assumptions of Subsection 2.1 and Hypotheses 1, 2 and 3 be satisfied for some $p \geqslant 2$. Then for any Lipschitz function $h: M \rightarrow \mathbb{R}, x_{0} \in M$ and for all $T^{\cdot}:[0,1] \rightarrow[1, \infty)$ satisfying $\varepsilon T^{\varepsilon} \rightarrow 0$ there exist positive constants $\varepsilon_{0} \in(0,1], k_{1}, k_{2}, k_{3}>0$ such that $\varepsilon \in\left(0, \varepsilon_{0}\right]$ implies

$$
\left(\mathbb{E}\left[\sup _{t \in[0, T]}\left|h\left(X_{t}^{\varepsilon}\left(x_{0}\right)\right)-h\left(X_{t}\left(x_{0}\right)\right)\right|^{p}\right]\right)^{\frac{1}{p}} \leqslant k_{1} \varepsilon^{\frac{p-1}{p^{2}}} \exp \left(k_{2} T\right) .
$$

In addition, the constant $k_{1}\left(x_{0}\right) \leqslant k_{3}\left(1+\bar{\eta}\left(x_{0}\right)\right)$.

We apply this result for the following setting.

Corollary 3.2 Let the assumptions of Proposition 3.1 be satisfied for some $p \geqslant 2$. Then for any $\lambda \in\left(0, \frac{p-1}{p^{2}}\right)$ there exist positive constants $c_{\lambda}, \varepsilon_{0} \in(0,1], k_{4}, k_{5}>0$ such that for $T_{\varepsilon}:=c_{\lambda}|\ln (\varepsilon)|$, $\varepsilon \in\left(0, \varepsilon_{0}\right]$ satisfies

$$
\left(\mathbb{E}\left[\sup _{t \in\left[0, T_{\varepsilon}\right]}\left|h\left(X_{t}^{\varepsilon}\left(x_{0}\right)\right)-h\left(X_{t}\left(x_{0}\right)\right)\right|^{p}\right]\right)^{\frac{1}{p}} \leqslant k_{4} \varepsilon^{\lambda} .
$$

In addition, the constant $k_{4}=k_{5} k_{1}$.

Proof: Plugging $T_{\varepsilon}=-c \ln (\varepsilon)$ in the right-hand side of (11) we obtain $k_{1} \varepsilon \exp \left(k_{2} T_{\varepsilon}\right)=k_{1} \varepsilon^{\frac{p-1}{p^{2}}-c k_{2}}$. Given $\lambda \in\left(0, \frac{p-1}{p^{2}}\right)$ we fix $c_{\lambda}:=\frac{1}{k_{2}}\left(\frac{p-1}{p^{2}}-\lambda\right)$ and infer the desired result.

The proof of Proposition 3.1 relies on the following lemma on positive invariant dynamical systems and the nonlinear comparison principle Corollary 5.2 given in the appendix. The main difficulty stems from the fact that the influence of the horizontal component in the vertical component cannot be estimated uniformly by the "diameter" of the leaf but has to be taken fully into account, which leads to a non-linear comparison principle.

Lemma 3.3 For $F \in \mathcal{C}^{2}\left(\mathbb{R}^{r+n}, L\left(\mathbb{R}^{r}, \mathbb{R}^{r+n}\right)\right)$ being a globally Lipschitz continuous matrix-valued vector field and $z \in \mathbb{R}^{r}$ denote by $(Y(t ; x, F z))_{t \geqslant 0}$ the unique global strong solution of the ordinary differential equation

$$
\frac{d Y}{d t}=F(Y) z \quad Y(0, x, F z)=x \in \mathbb{R}^{r+n} .
$$

1) Then there exists $C>0$ such that for any $z \in \mathbb{R}^{r}$ and $x, y \in M$ with $Y(t ; x)=Y(t ; x, F z)$ we have

$$
\sup _{t \geqslant 0}|(D F(Y(t ; x)) z) F(Y(t ; x)) z-(D F(Y(t ; y)) z) F(Y(t ; y)) z| \leqslant C|x-y|\|z\|^{2} .
$$

2) For any $x \in M$ we have $\sup _{t \in[0,1]}\|D F(Y(t ; x)) F(Y(t ; x))\|<\infty$.

A proof is given in [7] under Lemma 3.1.

Proof: (of Proposition 3.1) The first step of the proof yields the local orthogonality of the foliations and a transversal component by an appropriate change of coordinates. In a second step we estimate the transversal components with the help of the ergodic convergence of Hypothesis 3 and the nonlinear comparison principle Corollary 5.2. This is followed by the estimate of the horizontal component as the result of a classical Gronwall estimate before we conclude. 
1. Change of coordinates: We first rewrite $X^{\varepsilon}$ and $X$, the solutions of equations (11) and (5)), in terms of the coordinates given by the diffeomorphism $\varphi$

$$
\left(u_{t}, v_{t}\right):=\varphi\left(X_{t}\right) \quad \text { and } \quad\left(u_{t}^{\varepsilon}, v_{t}^{\varepsilon}\right):=\varphi\left(X_{t}^{\varepsilon}\right)
$$

The Lipschitz regularities of $h$ and $\varphi$ yields for $C_{0}:=\operatorname{Lip}\left(h \circ \varphi^{-1}\right)$ the estimate

$$
\left|h\left(X_{t}^{\varepsilon}\right)-h\left(X_{t}\right)\right| \leqslant C_{0}\left(\left|u_{t}^{\varepsilon}-u_{t}\right|+\left|v_{t}^{\varepsilon}-v_{t}\right|\right) .
$$

The proof of the statement consists in calculating estimates for each summand on the right hand side of equation above. We define the

$$
\begin{array}{rlrl}
\mathfrak{F}_{0} & :=(D \varphi) \circ F_{0} \circ \varphi^{-1}, & & \mathfrak{F}:=(D \varphi) \circ F \circ \varphi^{-1}, \\
\mathfrak{K}:=(D \varphi) \circ K \circ \varphi^{-1}, & & \tilde{\mathfrak{K}}:=(D \varphi) \circ \tilde{K} \circ \varphi^{-1},
\end{array}
$$

whose derivatives are uniformly bounded. Considering the components in the image of $\varphi$ we have:

$$
\mathfrak{K}=\left(\mathfrak{K}_{H}, \mathfrak{K}_{V}\right), \quad \tilde{\mathfrak{K}}=\left(\tilde{\mathfrak{K}}_{H}, \tilde{\mathfrak{K}}_{V}\right)
$$

with $\mathfrak{K}_{H}, \tilde{\mathfrak{K}}_{H} \in T L_{x_{0}}$ and $\mathfrak{K}_{V}, \tilde{\mathfrak{K}}_{V} \in T V \simeq \mathbb{R}^{d}$. The chain rule of the canonical Marcus equations mentioned in the introduction (Theorem 4.2 of [18]) yields for equation (5) the following form in $\varphi$ coordinates

$$
\begin{array}{rrr}
d u_{t}^{\varepsilon} & =\mathfrak{F}_{0}\left(u_{t}^{\varepsilon}, v_{t}^{\varepsilon}\right) d t+\mathfrak{F}\left(u_{t}^{\varepsilon}, v_{t}^{\varepsilon}\right) \diamond d Z_{t}+\varepsilon \mathfrak{K}_{H}\left(u_{t}^{\varepsilon}, v_{t}^{\varepsilon}\right) d t+\varepsilon \tilde{\mathfrak{K}}_{H}\left(v_{t}^{\varepsilon}\right) \diamond d \tilde{Z}_{t} & \text { with } u_{t}^{\varepsilon} \in L_{x_{0}}, \\
d v_{t}^{\varepsilon}=\varepsilon \mathfrak{K}_{V}\left(u_{t}^{\varepsilon}, v_{t}^{\varepsilon}\right) d t+\varepsilon \tilde{\mathfrak{K}}_{V}\left(v_{t}^{\varepsilon}\right) \diamond d \tilde{Z}_{t} & \text { with } v_{t}^{\varepsilon} \in V .
\end{array}
$$

2. Estimate of the transversal coordinate $\mathbb{E}\left[\sup \left|v^{\varepsilon}-v\right|^{p}\right]$ : Identically to [7], we start with estimates on the transversal components $\left|v^{\varepsilon}-v\right|$. The change of variables formula $x \mapsto g(x):=$ $|x|^{p}, x \in \mathbb{R}^{n+d}$ using $\langle D g(x), u\rangle=p|x|^{p-2}\langle x, u\rangle$ yields almost surely for $t \geqslant 0$

$$
\begin{aligned}
\left|v_{t}^{\varepsilon}-v_{t}\right|^{p}= & p \int_{0}^{t}\left|v_{s}^{\varepsilon}-v_{s}\right|^{p-2}\left\langle v_{s}^{\varepsilon}-v_{s}, \varepsilon \mathfrak{K}_{V}\left(u_{s}^{\varepsilon}, v_{s}^{\varepsilon}\right)\right\rangle d s \\
& +p \int_{0}^{t}\left|v_{s-}^{\varepsilon}-v_{s-}\right|^{p-2}\left\langle v_{s-}^{\varepsilon}-v_{s-}, \varepsilon \tilde{\mathfrak{K}}_{V}\left(v_{s-}^{\varepsilon}\right) \diamond d \tilde{Z}_{s}\right\rangle \\
\leqslant & p \int_{0}^{t}\left|v_{s}^{\varepsilon}-v_{s}\right|^{p-1}\left|\varepsilon \mathfrak{K}_{V}\left(u_{s}^{\varepsilon}, v_{s}^{\varepsilon}\right)-\varepsilon \mathfrak{K}_{V}\left(u_{s}, v_{s}\right)\right| d s \\
& +p \int_{0}^{t}\left|v_{s}^{\varepsilon}-v_{s}\right|^{p-1}\left|\varepsilon \mathfrak{K}_{V}\left(u_{s}, v_{s}\right)\right| d s \\
& +p \int_{0}^{t}\left|v_{s-}^{\varepsilon}-v_{s-}\right|^{p-2}\left|\left\langle v_{s-}^{\varepsilon}-v_{s-}, \varepsilon\left(\tilde{\mathfrak{K}}_{V}\left(v_{s-}^{\varepsilon}\right)-\tilde{\mathfrak{K}}_{V}\left(v_{s-}\right)\right) d \tilde{Z}_{s}\right\rangle\right| \\
& +p \int_{0}^{t}\left|v_{s-}^{\varepsilon}-v_{s-}\right|^{p-2}\left|\left\langle v_{s-}^{\varepsilon}-v_{s-}, \varepsilon \tilde{\mathfrak{K}}_{V}\left(v_{s-}\right) d \tilde{Z}_{s}\right\rangle\right| \\
& +p \sum_{0<s \leqslant t}\left|v_{s-}^{\varepsilon}-v_{s-}\right|^{p-1} \mid \Phi^{\varepsilon \tilde{\mathfrak{K}}_{V} \Delta_{s} \tilde{Z}}\left(v_{s-}^{\varepsilon}\right)-\Phi^{\varepsilon \tilde{\mathfrak{K}}_{V} \Delta_{s}} \tilde{Z}\left(v_{s-}\right) \\
& -\left(v_{s-}^{\varepsilon}-v_{s-}\right)-\varepsilon\left(\tilde{\mathfrak{K}}_{V}\left(v_{s-}^{\varepsilon}\right)-\varepsilon \tilde{\mathfrak{K}}_{V}\left(v_{s-}\right)\right) \Delta_{s} \tilde{Z} \mid \\
& +p \sum_{0<s \leqslant t}\left|v_{s-}^{\varepsilon}-v_{s-}\right|^{p-1}\left|\Phi^{\varepsilon \tilde{\mathfrak{K}}_{V} \Delta_{s} \tilde{Z}}\left(v_{s-}\right)-v_{s-}-\varepsilon \tilde{\mathfrak{K}}_{V}\left(v_{s-}\right) \Delta_{s} \tilde{Z}\right| \\
= & H_{1}+H_{2}+H_{3}+H_{4}+H_{5}+H_{6} .
\end{aligned}
$$


2.1 Pathwise estimates: $\mathbf{H}_{\mathbf{1}}$ : Clearly we have

$$
H_{1} \leqslant \varepsilon p \ell \int_{0}^{t}\left|v_{s}^{\varepsilon}-v_{s}\right|^{p} d s
$$

$\mathbf{H}_{2}$ : Young's inequality for the conjugate indices $p$ and $p /(p-1)$ yields

$$
\begin{aligned}
H_{2} & =\varepsilon p \int_{0}^{t}\left|v_{s}^{\varepsilon}-v_{s}\right|^{p-1}\left|\mathfrak{K}_{V}\left(u_{s}, v_{s}\right)\right| d s \\
& \leqslant \varepsilon p \sup _{[0, t]}\left|v^{\varepsilon}-v\right|^{p-1} \int_{0}^{t}\left|\mathfrak{K}_{V}\left(u_{s}, v_{s}\right)\right| d s \\
& \leqslant \varepsilon \sup _{[0, t]}\left|v^{\varepsilon}-v\right|^{p}+\varepsilon(p-1) t^{p}\left(\frac{1}{t} \int_{0}^{t}\left|\mathfrak{K}_{V}\left(u_{s}, v_{s}\right)\right| d s\right)^{p} .
\end{aligned}
$$

$\mathbf{H}_{3}$ and $\mathbf{H}_{4}$ : Switching to the Poisson random measure representation with respect to the compensated $\tilde{N}^{\prime}$, for instance see Kunita [20], we obtain

$$
H_{3} \leqslant \varepsilon p \int_{0}^{t} \int_{\mathbb{R}^{r}}\left|v_{s-}^{\varepsilon}-v_{s-}\right|^{p-2}\left\langle v_{s-}^{\varepsilon}-v_{s-},\left(\tilde{\mathfrak{K}}_{V}\left(v_{s-}^{\varepsilon}\right)-\tilde{\mathfrak{K}}_{V}\left(v_{s-}\right)\right) z\right\rangle \tilde{N}^{\prime}(d s d z)+\varepsilon C_{1} \int_{0}^{t}\left|v_{s}^{\varepsilon}-v_{s}\right|^{p} d s .
$$

and

$$
H_{4} \leqslant \varepsilon p \int_{0}^{t} \int_{\mathbb{R}^{r}}\left|v_{s-}^{\varepsilon}-v_{s-}\right|^{p-2}\left|\left\langle v_{s-}^{\varepsilon}-v_{s-}, \tilde{\mathfrak{K}}_{V}\left(v_{s-}\right) z\right\rangle\right| \tilde{N}^{\prime}(d s d z)+\varepsilon C_{2} \int_{0}^{t}\left|v_{s}^{\varepsilon}-v_{s}\right|^{p} d s .
$$

$\mathbf{H}_{\mathbf{5}}$ : For the canonical Marcus terms we apply Lemma 3.3, statement 1) which yields a positive constant such that

$$
H_{5} \leqslant \varepsilon^{2} C_{3} \int_{0}^{t} \int_{\mathbb{R}^{r}}\left|v_{s-}^{\varepsilon}-v_{s-}\right|^{p}\|z\|^{2} \tilde{N}^{\prime}(d s d z)+\varepsilon^{2} C_{4} \int_{0}^{t}\left|v_{s}^{\varepsilon}-v_{s}\right|^{p} d s .
$$

The details can be found in [7].

$\mathbf{H}_{6}$ : For the last term we apply Lemma 3.3, statement 2), and exploit that $\int_{\|z\|>1}\|z\|^{4} \nu^{\prime}(d z)<\infty$, we obtain a positive constant $C_{5}$ such that

$$
H_{5} \leqslant \varepsilon^{2} C_{5} \int_{0}^{t} \int_{\mathbb{R}^{r}}\left|v_{s-}^{\varepsilon}-v_{s-}\right|^{p-1}\|z\|^{4} \tilde{N}^{\prime}(d s d z)+\varepsilon^{2} C_{6} \int_{0}^{t}\left|v_{s}^{\varepsilon}-v_{s}\right|^{p-1} d s .
$$

Combining the estimates (17- 22) we obtain

$$
\begin{aligned}
\left|v_{t}^{\varepsilon}-v_{t}\right|^{p} \leqslant & \varepsilon \sup _{[0, t]}\left|v^{\varepsilon}-v\right|^{p}+\varepsilon(p-1) t^{p}\left(\frac{1}{t} \int_{0}^{t}\left|\mathfrak{K}_{V}\left(u_{s}, v_{s}\right)\right| d s\right)^{p} \\
& +\varepsilon\left(C_{1}+C_{2}\right) \int_{0}^{t}\left|v_{s}^{\varepsilon}-v_{s}\right|^{p} d s \\
& +\varepsilon^{2} C_{4} \int_{0}^{t}\left|v_{s}^{\varepsilon}-v_{s}\right|^{p} d s+\varepsilon^{2} C_{6} \int_{0}^{t}\left|v_{s}^{\varepsilon}-v_{s}\right|^{p-1} d s \\
& +\varepsilon p \int_{0}^{t} \int_{\mathbb{R}^{r}}\left|v_{s-}^{\varepsilon}-v_{s-}\right|^{p-2}\left\langle v_{s-}^{\varepsilon}-v_{s-}, \varepsilon \tilde{\mathfrak{K}}_{V}\left(v_{s-}\right) z\right\rangle \mid \tilde{N}^{\prime}(d s d z) \\
& +\varepsilon^{2} C_{3} \int_{0}^{t} \int_{\mathbb{R}^{r}}\left|v_{s-}^{\varepsilon}-v_{s-}\right|^{p}\|z\|^{2} \tilde{N}^{\prime}(d s d z) \\
& +\varepsilon^{2} p C_{5} \int_{0}^{t} \int_{\mathbb{R}^{r}}\left|v_{s-}^{\varepsilon}-v_{s-}\right|^{p-1}\|z\|^{4} \tilde{N}^{\prime}(d s d z) .
\end{aligned}
$$


2.2 Estimates on average: The main difference to [7] is found in the treatment of term $H_{2}$. In the sequel we drop the superscript of $T=T^{\varepsilon}$ where $T^{\varepsilon} \in[1, \infty)$ satisfying $\varepsilon T^{\varepsilon} \rightarrow 0$. Taking the supremum $t \in[0, T]$ and taking the expectation yields that the term (23) can be bounded by

$$
\varepsilon \mathbb{E}\left[\sup _{[0, T]}\left|v^{\varepsilon}-v\right|^{p}\right]+\varepsilon(p-1) C_{\infty} T^{p},
$$

where

$$
C_{\infty}=C_{\infty}\left(x_{0}\right)=\sup _{t \geqslant 0} \mathbb{E}\left[\left(\frac{1}{t} \int_{0}^{t}\left|\mathfrak{K}_{V}\left(u_{s}\left(x_{0}\right), 0\right)\right| d s\right)^{p}\right]<\infty
$$

due to the convergence

$$
\mathbb{E}\left[\left(\frac{1}{t} \int_{0}^{t}\left|\mathfrak{K}_{V}\left(u_{s}\left(x_{0}\right), 0\right)\right| d s-\int\left|\mathfrak{K}_{V}(y, 0)\right| \mu_{x_{0}}(d y)\right)^{p}\right] \rightarrow 0, \quad \text { as } t \rightarrow \infty .
$$

This implies in particular that

$$
C_{\infty}\left(x_{0}\right) \leqslant \int\left|\mathfrak{K}_{V}(y, 0)\right| \mu_{x_{0}}(d y)+\eta^{0}(0) \bar{\eta}\left(x_{0}\right) .
$$

We obtain the integral inequality

$$
\begin{aligned}
\mathbb{E}\left[\sup _{[0, T]}\left|v^{\varepsilon}-v\right|^{p}\right] \leqslant & \varepsilon C_{7} \mathbb{E}\left[\sup _{[0, T]}\left|v^{\varepsilon}-v\right|^{p}\right]+\varepsilon(p-1) C_{\infty} T^{p}+\varepsilon C_{8} \int_{0}^{T} \mathbb{E}\left[\sup _{[0, s]}\left|v^{\varepsilon}-v\right|^{p}\right] d s \\
& +\varepsilon C_{9} \int_{0}^{T} \mathbb{E}\left[\sup _{[0, s]}\left|v^{\varepsilon}-v\right|^{p-1}\right] d s \\
\leqslant & \varepsilon \mathbb{E}\left[\sup _{[0, T]}\left|v^{\varepsilon}-v\right|^{p}\right]+\varepsilon(p-1) C_{\infty} T^{p}+\varepsilon C_{8} \int_{0}^{T} \mathbb{E}\left[\sup _{[0, s]}\left|v^{\varepsilon}-v\right|^{p}\right] d s \\
& +\varepsilon C_{9} \int_{0}^{T} \mathbb{E}\left[\sup _{[0, s]}\left|v^{\varepsilon}-v\right|^{p}\right]^{\frac{p-1}{p}} d s .
\end{aligned}
$$

Hence for any value $\varepsilon \in\left(0, \frac{1}{2}\right]$ we eliminate the first term

$$
\begin{gathered}
\mathbb{E}\left[\sup _{[0, T]}\left|v^{\varepsilon}-v\right|^{p}\right] \leqslant 2 \varepsilon(p-1) C_{\infty} T^{p}+2 \varepsilon C_{8} \int_{0}^{T} \mathbb{E}\left[\sup _{[0, s]}\left|v^{\varepsilon}-v\right|^{p}\right] d s \\
+2 \varepsilon C_{9} \int_{0}^{T} \mathbb{E}\left[\sup _{[0, s]}\left|v^{\varepsilon}-v\right|^{p}\right]^{\frac{p-1}{p}} d s .
\end{gathered}
$$

That is, for $\Psi(T)=\mathbb{E}\left[\sup _{[0, T]}\left|v^{\varepsilon}-v\right|^{p}\right]$ we have

$$
\Psi(T) \leqslant \varepsilon C_{10} T^{p}+\varepsilon C_{11} \int_{0}^{T} \Psi(s) d s+\varepsilon C_{12} \int_{0}^{T} \Psi(s)^{\frac{p-1}{p}} d s .
$$

Using the nonlinear extension of the Gronwall-Bihari inequality in Corollary 5.2 in the appendix essentially given by Pachpatte [24], Theorem 2.4.2, which we adapt to our case we obtain a global constant $C>0$ such that using that $\varepsilon_{0} T$ is sufficiently small implies for all $\varepsilon \in\left(0, \varepsilon_{0}\right]$

$$
\Psi(T) \leqslant C\left(\varepsilon T^{p}+\varepsilon^{\frac{p-1}{p}} T^{p+\frac{p-1}{p}}\right) .
$$


3. Estimate of the horizontal component $\mathbb{E}\left[\sup \left|u^{\varepsilon}-u\right|^{p}\right]$ : $\quad$ For convenience of notation we restart with the numbering of constants. Formally we obtain

$$
\begin{aligned}
u_{t}^{\varepsilon}-u_{t}= & \int_{0}^{t}\left(\mathfrak{F}_{0}\left(u_{s}^{\varepsilon}, v_{s}^{\varepsilon}\right)-\mathfrak{F}_{0}\left(u_{s}, v_{s}\right)\right) d s+\int_{0}^{t}\left(\mathfrak{F}\left(u_{s-}^{\varepsilon}, v_{s-}^{\varepsilon}\right)-\mathfrak{F}\left(u_{s-}, v_{s-}\right)\right) \diamond d Z_{s} \\
& \quad+\varepsilon \int_{0}^{t}\left(\mathfrak{K}_{H}\left(u_{s}^{\varepsilon}, v_{s}^{\varepsilon}\right)-\mathfrak{K}_{H}\left(u_{s}, v_{s}\right)\right) d s+\varepsilon \int_{0}^{t} \mathfrak{K}_{H}\left(u_{s}, v_{s}\right) d s+\varepsilon \int_{0}^{t} \tilde{\mathfrak{K}}_{H}\left(v_{s-}^{\varepsilon}\right) \diamond d \tilde{Z}_{s} .
\end{aligned}
$$

For further details consult [7] where we obtain with the help of the change of variable formula for (30) the following equality in $\mathbb{R}^{n}$ almost surely for $t \geqslant 0$

$$
\begin{aligned}
\left|u_{t}^{\varepsilon}-u_{t}\right|^{p}= & p \int_{0}^{t}\left|u_{s}^{\varepsilon}-u_{s}\right|^{p-2}\left\langle u_{s}^{\varepsilon}-u_{s}, \mathfrak{F}_{0}\left(u_{s}^{\varepsilon}, v_{s}^{\varepsilon}\right)-\mathfrak{F}_{0}\left(u_{s}, v_{s}\right)\right\rangle d s \\
& +p \int_{0}^{t}\left|u_{s-}^{\varepsilon}-u_{s-}\right|^{p-2}\left\langle u_{s-}^{\varepsilon}-u_{s-},\left(\mathfrak{F}\left(u_{s-}^{\varepsilon}, v_{s-}^{\varepsilon}\right)-\mathfrak{F}\left(u_{s-}, v_{s-}\right)\right) d Z_{s}\right\rangle \\
& +p \sum_{0<s \leqslant t}\left|u_{s-}^{\varepsilon}-u_{s-}\right|^{p-2}\left\langle u_{s-}^{\varepsilon}-u_{s-}, \Phi^{\mathfrak{F} \Delta_{s} Z}\left(u_{s-}^{\varepsilon}, v_{s-}^{\varepsilon}\right)-\Phi^{\mathfrak{F} \Delta_{s} Z}\left(u_{s-}, v_{s-}\right)\right. \\
& +\varepsilon p \int_{0}^{t}\left|u_{s}^{\varepsilon}-u_{s}\right|^{p-2}\left\langle u_{s-}^{\varepsilon}-u_{s}, \mathfrak{K}_{H}\left(u_{s}^{\varepsilon}, v_{s}^{\varepsilon}\right)-\mathfrak{K}_{H}\left(u_{s}, v_{s}\right)\right\rangle d s \\
& +\varepsilon p \int_{0}^{t}\left|u_{s}^{\varepsilon}-u_{s}\right|^{p-2}\left\langle u_{s}^{\varepsilon}-u_{s}, \mathfrak{K}_{H}\left(u_{s}, v_{s}\right)\right\rangle d s \\
& +\varepsilon p \int_{0}^{t}\left|u_{s-}^{\varepsilon}-u_{s-}\right|^{p-2}\left\langle u_{s-}^{\varepsilon}-u_{s-}, \tilde{\mathfrak{K}}_{H}\left(v_{s-}^{\varepsilon}\right) d \tilde{Z}_{s}\right\rangle \\
& +p \sum_{0<s \leqslant t}\left|u_{s-}^{\varepsilon}-u_{s-}\right|^{p-2}\left\langle u_{s-}^{\varepsilon}-u_{s-}, \Phi^{\varepsilon \tilde{\mathfrak{K}}_{H} \Delta_{s}} \tilde{Z}_{\left(v_{s-}^{\varepsilon}\right.}^{\varepsilon}\right)-\Phi^{\varepsilon \tilde{\mathfrak{K}}_{H} \Delta_{s} \tilde{Z}}\left(v_{s-}\right) \\
& \left.-\left(v_{s-}^{\varepsilon}-v_{s-}\right)-\varepsilon\left(\tilde{\mathfrak{K}}_{H}\left(v_{s-}^{\varepsilon}\right)-\tilde{\mathfrak{K}}_{H}\left(v_{s-}\right)\right) \Delta_{s} \tilde{Z}\right\rangle \\
& \left.+p \sum_{0<s \leqslant t}\left|u_{s-}^{\varepsilon}-u_{s-}\right|^{p-2}\left\langle u_{s-}^{\varepsilon}-u_{s-}, \Phi^{\varepsilon \tilde{\mathfrak{K}}_{H} \Delta_{s}} \tilde{Z}_{\left(v_{s-}\right.}\right)-v_{s-}-\varepsilon \tilde{\mathfrak{K}}_{H}\left(v_{s-}\right) \Delta_{s} \tilde{Z}\right\rangle \\
= & I_{1}+I_{2}+I_{3}+I_{4}+I_{5}+I_{6}+I_{7}+I_{8} .
\end{aligned}
$$

In fact, we shall use the following estimate

$$
\left|u_{t}^{\varepsilon}-u_{t}\right|^{2 p} \leqslant 8^{p-1} \sum_{i=1}^{8} I_{i}^{2} .
$$

Now, we estimate each of the eight preceding summands on the right-hand side. The estimates of $I_{1}$ and $I_{4}$ are direct Lipschitz estimates. For the stochastic Itô terms we use the different kinds of maximal inequalities, see for instance 22 and 20. The estimate of the canonical Marcus terms $I_{3}, I_{7}$ and $I_{8}$ is the most difficult task in which we use the result of Lemma 3.3. The term $I_{5}$ is straightforward.

3.1 Estimate of the stochastic Itô integral terms $I_{2}$ and $I_{6}: \quad \mathbf{I}_{\mathbf{2}}$ : Due to the existence of moments of order at least $1, I_{2}$ has the following representation with respect to the compensated Poisson random measure associated to $Z$

$$
\int_{0}^{t}\left|u_{s-}^{\varepsilon}-u_{s-}\right|^{p-2}\left\langle u_{s-}^{\varepsilon}-u_{s-},\left(\mathfrak{F}\left(u_{s-}^{\varepsilon}, v_{s-}^{\varepsilon}\right)-\mathfrak{F}\left(u_{s-}, v_{s-}\right)\right) d Z_{s}\right\rangle
$$




$$
\begin{aligned}
=\int_{0}^{t} \int_{\mathbb{R}^{r}}\left|u_{s-}^{\varepsilon}-u_{s-}\right|^{p-2}\left\langle u_{s-}^{\varepsilon}-u_{s-},\left(\mathfrak{F}\left(u_{s-}^{\varepsilon}, v_{s-}^{\varepsilon}\right)-\mathfrak{F}\left(u_{s-}, v_{s-}\right)\right) z\right\rangle \tilde{N}(d s d z) \\
\quad+\int_{0}^{t} \int_{\|z\|>1}\left|u_{s}^{\varepsilon}-u_{s}\right|^{p-2}\left\langle u_{s}^{\varepsilon}-u_{s},\left(\mathfrak{F}\left(u_{s}^{\varepsilon}, v_{s}^{\varepsilon}\right)-\mathfrak{F}\left(u_{s}, v_{s}\right)\right) z\right\rangle \nu(d z) d s .
\end{aligned}
$$

For the first term (33) we exploit the embedding $L^{2} \subset L^{1}$, Kunita's maximal inequality (see [2] or [20]) for exponent equal to 2, and the Young inequality for the exponents $p / 2$ and $p /(p-2)$ combined with inequality (29) and obtain

$$
\begin{aligned}
& \mathbb{E}\left[\sup _{t \in[0, T]}\left|\int_{0}^{t} \int_{\mathbb{R}^{r}}\right| u_{s-}^{\varepsilon}-\left.u_{s-}\right|^{p-2}\left\langle u_{s-}^{\varepsilon}-u_{s-},\left(\mathfrak{F}\left(u_{s-}^{\varepsilon}, v_{s-}^{\varepsilon}\right)-\mathfrak{F}\left(u_{s-}, v_{s-}\right)\right) z\right\rangle \tilde{N}(d s d z) \mid\right]^{2} \\
& \leqslant \mathbb{E}\left[\sup _{t \in[0, T]}\left|\int_{0}^{t} \int_{\mathbb{R}^{r}}\right| u_{s-}^{\varepsilon}-\left.\left.u_{s-}\right|^{p-2}\left\langle u_{s-}^{\varepsilon}-u_{s-},\left(\mathfrak{F}\left(u_{s-}^{\varepsilon}, v_{s-}^{\varepsilon}\right)-\mathfrak{F}\left(u_{s-}, v_{s-}\right)\right) z\right\rangle \tilde{N}(d s d z)\right|^{2}\right] \\
& =\mathbb{E}\left[\int_{0}^{T} \int_{\mathbb{R}^{r}}\left|u_{s}^{\varepsilon}-u_{s}\right|^{2(p-2)}\left|\left\langle u_{s}^{\varepsilon}-u_{s},\left(\mathfrak{F}\left(u_{s}^{\varepsilon}, v_{s}^{\varepsilon}\right)-\mathfrak{F}\left(u_{s}, v_{s}\right)\right) z\right\rangle\right|^{2} \nu(d z) d s\right] \\
& \leqslant C_{1} \mathbb{E}\left[\int_{0}^{T} \int_{\mathbb{R}^{r}}\left|u_{s}^{\varepsilon}-u_{s}\right|^{2(p-1)}\left(\left|u_{s}^{\varepsilon}-u_{s}\right|^{2}+\left|v_{s}^{\varepsilon}-v_{s}\right|^{2}\right)\|z\|^{2} \nu(d z) d s\right] \\
& \leqslant C_{1}\left(\int_{\mathbb{R}^{r}}\|z\|^{2} \nu(d z)\right) \mathbb{E}\left[\int_{0}^{T}\left(\left|u_{s}^{\varepsilon}-u_{s}\right|^{2 p}+\left|u_{s}^{\varepsilon}-u_{s}\right|^{p-2}\left|v_{s}^{\varepsilon}-v_{s}\right|^{2}\right) d s\right] \\
& \leqslant C_{2}\left(\int_{0}^{T} \mathbb{E}\left[\sup _{[0, s]}\left|u^{\varepsilon}-u\right|^{2 p}\right] d s+\int_{0}^{T} \mathbb{E}\left[\left|v_{s}^{\varepsilon}-v_{s}\right|^{2 p}\right] d s\right) \\
& \leqslant C_{2}\left(\int_{0}^{T} \mathbb{E}\left[\sup _{[0, s]}\left|u^{\varepsilon}-u\right|^{2 p}\right] d s\right)+C\left(\varepsilon T^{2 p}+\varepsilon^{\frac{2 p-1}{2 p}} T^{2(p+1)+1}\right) .
\end{aligned}
$$

The second term follows directly by Young's inequality and the Lipschitz continuity of $\mathfrak{F}$

$$
\begin{aligned}
& \mathbb{E}\left[\sup _{t \in[0, T]} \int_{0}^{t} \int_{\|z\|>1}\left|u_{s}^{\varepsilon}-u_{s}\right|^{p-2}\left\langle u_{s}^{\varepsilon}-u_{s},\left(\mathfrak{F}\left(u_{s}^{\varepsilon}, v_{s}^{\varepsilon}\right)-\mathfrak{F}\left(u_{s}, v_{s}\right)\right) z\right\rangle \nu(d z) d s\right]^{2} \\
& \leqslant\left(\ell \int_{\|z\|>1}\|z\| \nu(d z) \mathbb{E}\left[\sup _{t \in[0, T]} \int_{0}^{t}\left(\left|u_{s}^{\varepsilon}-u_{s}\right|^{p}+\left|u_{s}^{\varepsilon}-u_{s}\right|^{p-1}\left|v_{s}^{\varepsilon}-v_{s}\right|\right) d s\right]\right)^{2} \\
& \leqslant\left(\ell \int_{\|z\|>1}\|z\| \nu(d z)\left(2 \int_{0}^{T} \mathbb{E}\left[\sup _{[0, s]}\left|u^{\varepsilon}-u\right|^{p}\right] d s+\int_{0}^{T} \mathbb{E}\left[\left|v_{s}^{\varepsilon}-v_{s}\right|^{p}\right] d s\right)\right)^{2} \\
& \leqslant C_{3} T \int_{0}^{T} \mathbb{E}\left[\sup _{[0, s]}\left|u^{\varepsilon}-u\right|^{2 p}\right] d s+C\left(\varepsilon T^{2 p}+\varepsilon^{\frac{2 p-1}{2 p}} T^{2(p+1)+1}\right) .
\end{aligned}
$$

$\mathbf{I}_{6}$ : We go over to the representation with the Poisson random measure $\tilde{N}^{\prime}$ associated to the Lévy process $\tilde{Z}$ and obtain

$$
\begin{aligned}
& \sup _{t \in[0, T]} \varepsilon \int_{0}^{t}\left|u_{s-}^{\varepsilon}-u_{s-}\right|^{p-2}\left\langle u_{s-}^{\varepsilon}-u_{s-}, \tilde{\mathfrak{K}}_{H}\left(v_{s-}^{\varepsilon}\right) d \tilde{Z}_{s}\right\rangle \\
& =\sup _{t \in[0, T]} \varepsilon \int_{0}^{t} \int_{\mathbb{R}^{r}}\left|u_{s-}^{\varepsilon}-u_{s-}\right|^{p-2}\left\langle u_{s-}^{\varepsilon}-u_{s-},\left(\tilde{\mathfrak{K}}_{H}\left(v_{s-}^{\varepsilon}\right)-\tilde{\mathfrak{K}}_{H}\left(v_{s-}\right)\right) z\right\rangle \tilde{N}^{\prime}(d s d z) \\
& \quad+\sup _{t \in[0, T]} \varepsilon \int_{0}^{t} \int_{\|z\|>1}\left|u_{s}^{\varepsilon}-u_{s}\right|^{p-2}\left\langle u_{s}^{\varepsilon}-u_{s},\left(\tilde{\mathfrak{K}}_{H}\left(v_{s}^{\varepsilon}\right)-\tilde{\mathfrak{K}}_{H}\left(v_{s}\right)\right) z\right\rangle \nu^{\prime}(d z) d s
\end{aligned}
$$




$$
\begin{aligned}
& +\sup _{t \in[0, T]} \varepsilon \int_{0}^{t} \int_{\mathbb{R}^{r}}\left|u_{s-}^{\varepsilon}-u_{s-}\right|^{p-2}\left\langle u_{s-}^{\varepsilon}-u_{s-}, \tilde{\mathfrak{K}}_{H}\left(v_{s-}\right) z\right\rangle \tilde{N}^{\prime}(d s d z) \\
& +\sup _{t \in[0, T]} \varepsilon \int_{0}^{t} \int_{\|z\|>1}\left|u_{s}^{\varepsilon}-u_{s}\right|^{p-2}\left\langle u_{s}^{\varepsilon}-u_{s}, \tilde{\mathfrak{K}}_{H}\left(v_{s}\right) z\right\rangle \nu^{\prime}(d z) d s .
\end{aligned}
$$

The terms $J_{1}$ and $J_{2}$ are estimated analogously to (35) and (36) where $\mathfrak{F}$ is replaced by $\tilde{\mathfrak{K}}_{H}$, which yield the following estimates

$$
\begin{aligned}
& \left(\mathbb{E}\left[\sup _{t \in[0, T]}\left|\varepsilon \int_{0}^{t} \int_{\mathbb{R}^{r}}\right| u_{s-}^{\varepsilon}-\left.u_{s-}\right|^{p-2}\left\langle u_{s-}^{\varepsilon}-u_{s-},\left(\tilde{\mathfrak{K}}_{H}\left(v_{s-}^{\varepsilon}\right)-\tilde{\mathfrak{K}}_{H}\left(v_{s-}\right)\right) z\right\rangle \tilde{N}^{\prime}(d s d z) \mid\right]\right)^{2} \\
& \leqslant C_{4}\left(\int_{0}^{T} \mathbb{E}\left[\sup _{[0, s]}\left|u^{\varepsilon}-u\right|^{2 p}\right] d s\right)^{\frac{1}{2}}+C\left(\varepsilon T^{2 p}+\varepsilon^{\frac{2 p-1}{2 p}} T^{2(p+1)+1}\right) \quad \text { and } \\
& \left(\mathbb{E}\left[\sup _{[0, T]} \varepsilon\left|\int_{0}^{t} \int_{\|z\|>1}\right| u_{s}^{\varepsilon}-\left.u_{s}\right|^{p-2}\left\langle u_{s}^{\varepsilon}-u_{s},\left(\tilde{\mathfrak{K}}_{H}\left(v_{s}^{\varepsilon}\right)-\tilde{\mathfrak{K}}_{H}\left(v_{s}\right)\right) z\right\rangle \nu^{\prime}(d z) d s \mid\right]\right)^{2} \\
& \leqslant C_{5} \int_{0}^{T} \mathbb{E}\left[\sup _{[0, s]}\left|u^{\varepsilon}-u\right|^{2 p}\right] d s+C\left(\varepsilon T^{2 p}+\varepsilon^{\frac{2 p-1}{2 p}} T^{2(p+1)+1}\right) .
\end{aligned}
$$

For the term $J_{3}$ we observe that $v_{s}=0$ consequently $\mathfrak{K}_{V}\left(v_{s}\right)$ is constant. Applying Kunita's maximal inequality for the exponent 2 , we obtain

$$
\begin{aligned}
& \left(\mathbb{E}\left[\sup _{t \in[0, T]} \varepsilon\left|\int_{0}^{t} \int_{\mathbb{R}^{r}}\right| u_{s-}^{\varepsilon}-\left.u_{s-}\right|^{p-2}\left\langle u_{s-}^{\varepsilon}-u_{s-}, \tilde{\mathfrak{K}}_{H}\left(v_{s-}\right) z\right\rangle \tilde{N}^{\prime}(d s d z) \mid\right]\right)^{2} \\
& \leqslant \varepsilon^{2} \mathbb{E}\left[\sup _{t \in[0, T]}\left|\int_{0}^{t} \int_{\mathbb{R}^{r}}\right| u_{s-}^{\varepsilon}-\left.\left.u_{s-}\right|^{p-2}\left\langle u_{s-}^{\varepsilon}-u_{s-}, \tilde{\mathfrak{K}}_{H}\left(v_{s-}\right) z\right\rangle \tilde{N}^{\prime}(d s d z)\right|^{2}\right] \\
& \leqslant \varepsilon^{2} C_{6} \int_{0}^{T} \int_{\mathbb{R}^{r}} \mathbb{E}\left[\left|u_{s}^{\varepsilon}-u_{s}\right|^{2(p-1)}\right]\|z\|^{2} \nu^{\prime}(d z) d s \\
& \leqslant \varepsilon^{2} C_{6}\left(\int_{\mathbb{R}^{r}}\|z\|^{2} \nu^{\prime}(d z)\right)\left(\int_{0}^{T} \mathbb{E}\left[\sup _{[0, s]}\left|u^{\varepsilon}-u\right|^{2 p-2}\right] d s\right) \\
& \leqslant \varepsilon^{2} C_{7} \int_{0}^{T} \mathbb{E}\left[\sup _{[0, s]}\left|u^{\varepsilon}-u\right|^{2 p-2}\right] d s \\
& \leqslant \varepsilon^{2} C_{7}\left(\int_{0}^{T} \mathbb{E}\left[\sup _{[0, s]}\left|u^{\varepsilon}-u\right|^{2 p}\right] d s+\frac{C_{8}}{p} T\right) .
\end{aligned}
$$

The term $J_{4}$ is again easier, using $\varepsilon T<1$ and $\varepsilon<1$ we obtain

$$
\begin{aligned}
& \left(\mathbb{E}\left[\sup _{[0, T]} \varepsilon \int_{0}^{t} \int_{\|z\|>1}\left|u_{s}^{\varepsilon}-u_{s}\right|^{p-2}\left\langle u_{s}^{\varepsilon}-u_{s}, \tilde{\mathfrak{K}}_{H}\left(v_{s}\right) z\right\rangle \nu^{\prime}(d z) d s\right]\right)^{2} \\
& \leqslant\left(\varepsilon \int_{\|z\|>1}\|z\| \nu^{\prime}(d z)\left\|\tilde{\mathfrak{K}}_{H}(0)\right\| \int_{0}^{T} \mathbb{E}\left[\sup _{[0, s]}\left|u^{\varepsilon}-u\right|^{p-1}\right] d s\right)^{2} \\
& \leqslant \varepsilon^{2} C_{9}\left(\int_{0}^{T} \mathbb{E}\left[\sup _{[0, s]}\left|u^{\varepsilon}-u\right|^{p}\right] d s\right)^{2}+C_{9} \varepsilon^{2 p} T^{2} \\
& \leqslant \varepsilon^{2} T C_{9} \int_{0}^{T} \mathbb{E}\left[\sup _{[0, s]}\left|u^{\varepsilon}-u\right|^{2 p}\right] d s+C_{9} \varepsilon^{2 p} T^{2}
\end{aligned}
$$




$$
\leqslant C_{9} \int_{0}^{T} \mathbb{E}\left[\sup _{[0, s]}\left|u^{\varepsilon}-u\right|^{2 p}\right] d s+C_{9} \varepsilon^{2 p} T^{2}
$$

Summing up we obtain

$$
\mathbb{E}\left[\sup _{[0, T]}\left|I_{6}\right|^{2}\right] \leqslant C_{10}\left(\int_{0}^{T} \mathbb{E}\left[\sup _{[0, s]}\left|u^{\varepsilon}-u\right|^{p}\right] d s+\varepsilon^{\frac{2 p-1}{2 p}} T^{2(p+1)+1}+\varepsilon^{2} T\right) .
$$

3.2 Estimate of the canonical Marcus terms $I_{3}, I_{7}$ and $I_{8}$ : The estimate is identical to estimate (54) in [7] and yields a constant $C_{11}$ such that

$$
\left|I_{3}\right| \leqslant 2 C_{11}\left(\sum_{0<s \leqslant t}\left|u_{s-}^{\varepsilon}-u_{s-}\right|^{p}\left\|\Delta_{s} Z\right\|^{2}+\sum_{0<s \leqslant t}\left|v_{s-}^{\varepsilon}-v_{s-}\right|^{p}\left\|\Delta_{s} Z\right\|^{2}\right) .
$$

Once again, the representation of this sum in terms of the Poisson random measure given in Kunita [20] tells us that

$$
\begin{aligned}
& \sum_{0<s \leqslant t}\left|u_{s-}^{\varepsilon}-u_{s-}\right|^{p}\left\|\Delta_{s} Z\right\|^{2} \\
& =\int_{0}^{t} \int_{\mathbb{R}^{r}}\left|u_{s-}^{\varepsilon}-u_{s-}\right|^{p}\|z\|^{2} \tilde{N}(d s d z)+\int_{0}^{t} \int_{\|z\|>1}\left|u_{s}^{\varepsilon}-u_{s}\right|^{p}\|z\|^{2} \nu(d z) d s .
\end{aligned}
$$

The maximal inequality for integrals with respect to the compensated Poisson random measures and inequality (29) yield

$$
\begin{aligned}
& \mathbb{E}\left[\sup _{[0, T]}\left|I_{3}\right|^{2}\right] \leqslant C_{12} \int_{0}^{T} \int_{\mathbb{R}^{r}}\left(\mathbb{E}\left[\sup _{[0, s]}\left|u^{\varepsilon}-u\right|^{2 p}\right]+\mathbb{E}\left[\left|v_{s}^{\varepsilon}-v_{s}\right|^{2 p}\right]\right)\|z\|^{4} \nu(d z) d s \\
& =C_{12} \int_{\mathbb{R}^{r}}\|z\|^{4} \nu(d z)\left(\int_{0}^{T}\left(\mathbb{E}\left[\sup _{[0, s]}\left|u^{\varepsilon}-u\right|^{2 p}\right]+\mathbb{E}\left[\left|v_{s}^{\varepsilon}-v_{s}\right|^{2 p}\right]\right) d s\right) \\
& \leqslant C_{13}\left(\int_{0}^{T} \mathbb{E}\left[\sup _{[0, s]}\left|u^{\varepsilon}-u\right|^{2 p} d s+C\left(\varepsilon T^{2 p}+\varepsilon^{\frac{2 p-1}{2 p}} T^{2(p+1)+1}\right)\right) .\right.
\end{aligned}
$$

$\mathbf{I}_{7}$ : For $I_{7}$ we apply Lemma 3.3 statement 1) and Young's inequality and obtain the analogous result

$$
\begin{aligned}
& \sum_{0<s \leqslant t}\left|u_{s-}^{\varepsilon}-u_{s-}\right|^{p-2}\left\langle u_{s-}^{\varepsilon}-u_{s-}, \Phi^{\varepsilon \tilde{\mathfrak{K}}_{H} \Delta_{s} \tilde{Z}}\left(v_{s-}^{\varepsilon}\right)-\Phi^{\varepsilon \tilde{\mathfrak{K}}_{H} \Delta_{s} \tilde{Z}}\left(v_{s-}\right)\right. \\
& \left.\quad-\left(v_{s-}^{\varepsilon}-v_{s-}\right)-\varepsilon\left(\tilde{\mathfrak{K}}_{H}\left(v_{s-}^{\varepsilon}\right)-\tilde{\mathfrak{K}}_{H}\left(v_{s-}\right)\right) \Delta_{s} \tilde{Z}\right\rangle \\
& \leqslant \varepsilon^{2} C_{14}\left(\sum_{0<s \leqslant t}\left(\left|u_{s-}^{\varepsilon}-u_{s-}\right|^{p}+\left|v_{s-}^{\varepsilon}-v_{s-}\right|^{p}\right)\left\|\Delta_{s} \tilde{Z}\right\|^{2}\right) .
\end{aligned}
$$

Rewriting the last expression in terms of the (compensated) Poisson random measure $\tilde{N}^{\prime}$ we obtain

$$
\begin{aligned}
& \sum_{0<s \leqslant t}\left(\left|u_{s-}^{\varepsilon}-u_{s-}\right|^{p}+\left|v_{s-}^{\varepsilon}-v_{s-}\right|^{p}\right)\left\|\Delta_{s} \tilde{Z}\right\|^{2} \\
& =\int_{0}^{t} \int_{\mathbb{R}^{r}}\left(\left|u_{s-}^{\varepsilon}-u_{s-}\right|^{p}+\left|v_{s-}^{\varepsilon}-v_{s-}\right|^{p}\right)\|z\|^{2} \tilde{N}^{\prime}(d s d z)
\end{aligned}
$$




$$
+\int_{0}^{t} \int_{\|z\|>1}\left(\left|u_{s}^{\varepsilon}-u_{s}\right|^{p}+\left|v_{s}^{\varepsilon}-v_{s}\right|^{p}\right)\|z\|^{2} \nu^{\prime}(d z) d s .
$$

Kunita's maximal inequality for the exponent 2 yields

$$
\begin{aligned}
& \mathbb{E}\left[\left|\sup _{[0, T]} \int_{0}^{t} \int_{\mathbb{R}^{r}}\left(\left|u_{s-}^{\varepsilon}-u_{s-}\right|^{p}+\left|v_{s-}^{\varepsilon}-v_{s-}\right|^{p}\right)\|z\|^{2} \tilde{N}^{\prime}(d s d z)\right|^{2}\right] \\
& \leqslant C_{15} \int_{0}^{T} \int_{\mathbb{R}^{r}} \mathbb{E}\left[\left|u_{s}^{\varepsilon}-u_{s}\right|^{2 p}+\left|v_{s}^{\varepsilon}-v_{s}\right|^{2 p}\right]\|z\|^{2} \nu^{\prime}(d z) d s \\
& \leqslant C_{16}\left(\int_{\mathbb{R}^{r}}\|z\|^{2} \nu^{\prime}(d z) \int_{0}^{T} \mathbb{E}\left[\sup _{[0, s]}\left|u^{\varepsilon}-u\right|^{2 p}\right] d s+\int_{0}^{T} \mathbb{E}\left[\left|v_{s}^{\varepsilon}-v_{s}\right|^{2 p}\right] d s\right) \\
& \leqslant C_{16} \int_{\mathbb{R}^{r}}\|z\|^{2} \nu^{\prime}(d z) \int_{0}^{T} \mathbb{E}\left[\sup _{[0, s]}\left|u^{\varepsilon}-u\right|^{2 p}\right] d s+C_{17}\left(\varepsilon T^{2 p}+\varepsilon^{\frac{2 p-1}{2 p}} T^{2(p+1)+1}\right),
\end{aligned}
$$

where $C_{17}=C$ from (29). The term (42) is treated obviously such that

$$
\mathbb{E}\left[\sup _{[0, T]}\left|I_{7}\right|^{2}\right] \leqslant \varepsilon^{2} C_{18} \int_{\mathbb{R}^{r}}\|z\|^{4} \nu^{\prime}(d z) \int_{0}^{T} \mathbb{E}\left[\sup _{[0, s]}\left|u^{\varepsilon}-u\right|^{2 p}\right] d s+C_{17}\left(\varepsilon T^{2 p}+\varepsilon^{\frac{2 p-1}{2 p}} T^{2(p+1)+1}\right) .
$$

I $_{8}$ : For $I_{8}$ Lemma 3.3, statement 2), yields

$$
\begin{aligned}
& \sum_{0<s \leqslant t}\left|u_{s-}^{\varepsilon}-u_{s-}\right|^{p-2}\left\langle u_{s-}^{\varepsilon}-u_{s-}, \Phi^{\varepsilon \tilde{\mathfrak{K}}_{H} \Delta_{s} \tilde{Z}}\left(v_{s-}\right)-v_{s-}-\varepsilon \tilde{\mathfrak{K}}_{H}\left(v_{s-}\right) \Delta_{s} \tilde{Z}\right\rangle \\
& \leqslant \sum_{0<s \leqslant t}\left|u_{s-}^{\varepsilon}-u_{s-}\right|^{p-1}\left|\Phi^{\varepsilon \tilde{\mathfrak{K}}_{H} \Delta_{s} \tilde{Z}}\left(v_{s-}\right)-v_{s-}-\varepsilon \tilde{\mathfrak{K}}_{H}\left(v_{s-}\right) \Delta_{s} \tilde{Z}\right| \\
& \leqslant \varepsilon^{2} C_{19} \sum_{0<s \leqslant t}\left|u_{s-}^{\varepsilon}-u_{s-}\right|^{p-1}\left\|\Delta_{s} \tilde{Z}\right\|^{2}
\end{aligned}
$$

such that again Kunita's inequality with exponent 2 and elementary Young's estimate for parameters $\frac{p-2}{p}$ and $\frac{p}{2}$ yield

$$
\begin{aligned}
\mathbb{E}\left[\sup _{[0, T]}\left|I_{8}\right|^{2}\right] & \leqslant \varepsilon^{2} C_{20} \int_{\mathbb{R}^{r}}\|z\|^{4} \nu^{\prime}(d z) \int_{0}^{T} \mathbb{E}\left[\sup _{[0, s]}\left|u^{\varepsilon}-u\right|^{2 p-2}\right] d s \\
& \leqslant \varepsilon C_{21} \int_{0}^{T} \mathbb{E}\left[\sup _{[0, s]}\left|u^{\varepsilon}-u\right|^{2 p}\right] d s+C_{21} \varepsilon^{\frac{p}{2}} T .
\end{aligned}
$$

\subsection{Estimate of $I_{5}$ :}

$$
\begin{aligned}
\int_{0}^{T}\left|u_{s}^{\varepsilon}-u_{s}\right|^{p-2}\left\langle u_{s}^{\varepsilon}-u_{s}, \varepsilon \mathfrak{K}_{H}\left(u_{s}, v_{s}\right)\right\rangle d s & \leqslant C_{22} \int_{0}^{T} \varepsilon\left|u_{s}^{\varepsilon}-u_{s}\right|^{p-1} d s \\
& \leqslant C_{22} \int_{0}^{T} \varepsilon\left|u_{s}^{\varepsilon}-u_{s}\right|^{p} d s+C_{22} \varepsilon^{p} T
\end{aligned}
$$

such that

$$
\mathbb{E}\left[\sup _{[0, T]}\left|I_{5}\right|^{2}\right] \leqslant \varepsilon C_{23} T \int_{0}^{T} \mathbb{E}\left[\sup _{[0, s]}\left|u^{\varepsilon}-u\right|^{2 p}\right] d s+C_{23} \varepsilon^{2 p} T^{2}
$$




\subsection{Linear comparison principle:}

Taking the supremum and the expectation of the left-hand side of equation (31) and combining the estimates of $8^{p-1} \sum_{i=1}^{8} \mathbb{E}\left[\sup _{[0, T]}\left|I_{i}\right|\right]$ given by (35), (36), (37), (40), (43), (44) and (45) we obtain a positive constant $C_{24}$

$$
\begin{aligned}
\mathbb{E}\left[\sup _{[0, T]}\left|u^{\varepsilon}-u\right|^{2 p}\right] & \leqslant C_{24}\left(\int_{0}^{T} \mathbb{E}\left[\sup _{[0, s]}\left|u^{\varepsilon}-u\right|^{2 p}\right] d s+\varepsilon T^{2 p}+\varepsilon^{\frac{2 p-1}{2 p}} T^{2(p+1)+1}+\left(\varepsilon^{p} T\right)^{2}\right) \\
& \leqslant C_{24}\left(\int_{0}^{T} \mathbb{E}\left[\sup _{[0, s]}\left|u^{\varepsilon}-u\right|^{2 p}\right] d s+\varepsilon T^{2 p+3}\right) .
\end{aligned}
$$

Finally

$$
\mathbb{E}\left[\sup _{[0, T]}\left|u^{\varepsilon}-u\right|^{p}\right] \leqslant \mathbb{E}\left[\sup _{[0, T]}\left|u^{\varepsilon}-u\right|^{2 p}\right] \leqslant C_{25} \varepsilon^{\frac{2 p-1}{2 p}} T^{2 p+3} e^{C_{25} T} .
$$

4. Conclusion: The estimates of the sum of the vertical and the horizontal estimate yield

$$
\begin{aligned}
\mathbb{E}\left[\sup _{[0, T]}\left|h\left(X^{\varepsilon}\right)-h(X)\right|^{p}\right] & \leqslant C_{0}\left(\mathbb{E}\left[\sup _{[0, T]}\left|u^{\varepsilon}-u\right|^{p}\right]+\mathbb{E}\left[\sup _{[0, T]}\left|v^{\varepsilon}-v\right|^{p}\right]\right) \\
& \leqslant C_{26} \varepsilon^{\frac{2 p-1}{2 p}} T^{2 p+3} e^{C_{25} T}+C\left(\varepsilon T^{p}+\varepsilon^{\frac{p-1}{p}} T^{p+\frac{p-1}{p}}\right) \\
& \leqslant C_{27} \varepsilon^{\frac{p-1}{p}} e^{C_{27} T}
\end{aligned}
$$

We finally note that the only dependence on the initial conditions stems from $C_{\infty}$ and hence by (28) the estimate $C_{27} \leqslant C_{28}\left(1+\eta^{0}(0) \bar{\eta}\left(x_{0}\right)\right)$. This finishes the proof.

\section{The averaging error and the proof of the main result}

For convenience we fix the following notation. Given $h: M \rightarrow \mathbb{R}^{n}$ a globally Lipschitz continuous function and $Q^{h}: V \rightarrow \mathbb{R}^{n}$ its average on the leaves defined in definition (6). For $t \geqslant 0, x_{0} \in M$ and $\varepsilon \in(0,1]$ we write

$$
\delta_{x_{0}}^{h}(\varepsilon, t):=\int_{0}^{t \wedge \varepsilon \tau^{\varepsilon}} h\left(X_{\frac{s}{\varepsilon}}^{\varepsilon}\left(x_{0}\right)\right)-Q^{h}\left(\pi\left(X_{\frac{s}{\varepsilon}}^{\varepsilon}\left(x_{0}\right)\right)\right) d s .
$$

Proposition 4.1 Let the assumptions of Proposition 3.1 be satisfied for fixed $p \geqslant 2$. Then for any globally Lipschitz continuous function $h: M \rightarrow \mathbb{R}^{n}, \lambda \in\left(0, \frac{p-1}{p^{2}}\right)$ and $x_{0} \in M$ there exist constants $b_{1}>0$ and $\varepsilon_{0} \in(0,1]$ such that for $\varepsilon \in\left(0, \varepsilon_{0}\right]$ and $T \in[0,1]$ we have

$$
\left(\mathbb{E}\left[\sup _{s \in[0, T]}\left|\delta_{x_{0}}^{h}(\varepsilon, s)\right|^{p}\right]\right)^{\frac{1}{p}} \leqslant b_{1} T\left[\varepsilon^{\lambda}+\eta^{0}(c T|\ln \varepsilon|)\right],
$$

where $c:=\frac{1}{k_{2}}\left(\frac{p-1}{p^{2}}-\lambda\right) \wedge \ell \operatorname{Lip}\left(\varphi^{-1}\right)$ is given in Corollary [3.2. and $\eta^{0}$ is the temporal factor of the ergodic rate of convergence given in equation (9) by Hypothesis 3. 
Proof of Proposition (4.1) : Fix $x_{0} \in M$. For $\varepsilon \in(0,1)$ and $T>0$ we define the partition

$$
t_{0}=0<t_{1}^{\varepsilon}<\cdots<t_{N^{\varepsilon}}^{\varepsilon} \leqslant \frac{T}{\varepsilon} \wedge \tau^{\varepsilon}
$$

with the following step size

$$
\Delta_{\varepsilon}:=-c T \ln (\varepsilon) \quad \text { for some } c>0 .
$$

The grid points of the partition are given by $t_{n}^{\varepsilon}:=n \Delta_{\varepsilon} \wedge \tau^{\varepsilon}$ for $0 \leqslant n \leqslant N_{\varepsilon}$ for $\varepsilon \in(0,1]$ with $N_{\varepsilon}=\left\lfloor\frac{1}{c \varepsilon \mid \ln (\varepsilon)\rfloor}\right\rfloor$. The term $\delta_{x_{0}}^{h}(\varepsilon, t)$ can be estimated by the following three sums

$$
\left|\delta_{x_{0}}^{h}(\varepsilon, T)\right| \leqslant\left|A_{1}(T, \varepsilon)\right|+\left|A_{2}(T, \varepsilon)\right|+\left|A_{3}(T, \varepsilon)\right|,
$$

where

$$
\begin{aligned}
& A_{1}(T, \varepsilon):=\varepsilon \sum_{n=0}^{N_{\varepsilon}} \int_{t_{n}}^{t_{n+1}}\left[h\left(X_{s}^{\varepsilon}\left(x_{0}\right)\right)-h\left(X_{s-t_{n}}\left(X_{t_{n}}^{\varepsilon}\left(x_{0}\right)\right)\right)\right] d s \\
& A_{2}(T, \varepsilon):=\varepsilon \sum_{n=0}^{N_{\varepsilon}} \int_{t_{n}}^{t_{n+1}}\left[h\left(X_{s-t_{n}}\left(X_{t_{n}}^{\varepsilon}\left(x_{0}\right)\right)\right)-\Delta_{\varepsilon} Q\left(\pi\left(X_{t_{n}}^{\varepsilon}\left(x_{0}\right)\right)\right)\right] d s, \\
& A_{3}(T, \varepsilon):=\sum_{n=0}^{N_{\varepsilon}} \varepsilon \Delta_{\varepsilon} Q\left(\pi\left(X_{t_{n}}^{\varepsilon}\left(x_{0}\right)\right)\right)-\int_{0}^{t_{N_{\varepsilon}+1}} \varepsilon Q\left(\pi\left(X_{\frac{s}{\varepsilon}}^{\varepsilon}\left(x_{0}\right)\right)\right) d s .
\end{aligned}
$$

The following lemmas estimate the preceding terms one-by-one. For convenience of the reader we number the constants $C_{i}$.

Lemma 4.2 For any $\lambda \in\left(0, \frac{p-1}{p}\right)$ there exist positive constants $b_{2}>0$ and $\varepsilon_{0} \in(0,1]$ such that for any $\varepsilon \in\left(0, \varepsilon_{0}\right]$ and $T \geqslant 0$

$$
\left(\mathbb{E}\left[\sup _{s \in[0, T]}\left|A_{1}(s, \varepsilon)\right|^{p}\right]\right)^{\frac{1}{p}} \leqslant b_{2} T \varepsilon^{\lambda} .
$$

Proof: Using the Markov property analogously to [7] and Corollary 3.2] we obtain

$$
\begin{aligned}
& \mathbb{E}\left[\left.\sup _{[0, T]} A_{1}(s, \varepsilon)\right|^{p}\right]^{\frac{1}{p}} \\
& =\varepsilon \sum_{n=0}^{N_{\varepsilon}-1} \mathbb{E}\left[\mathbb { E } \left[\left|\int_{t_{n}}^{t_{n+1}}\right| h\left(X_{s}^{\varepsilon}\left(x_{0}\right)-h\left(\left.X_{s-t_{n}}\left(X_{t_{n}}^{\varepsilon}\left(x_{0}\right)\right) d s\right|^{p} \mid \mathcal{F}_{t_{n}}\right]\right]^{\frac{1}{p}}\right.\right. \\
& =\varepsilon \sum_{n=0}^{N_{\varepsilon}-1} \mathbb{E}\left[\mathbb{E}\left[\left|\int_{t_{n}}^{t_{n+1}}\right| h\left(X_{s-t_{n}}^{\varepsilon}(y)\right)-h\left(\left.X_{s-t_{n}}(y) d s\right|^{p} \mid y=X_{t_{n}}^{\varepsilon}\left(x_{0}\right)\right]\right]^{\frac{1}{p}}\right. \\
& \leqslant \varepsilon\left(N_{\varepsilon}+1\right) \Delta_{\varepsilon} \max _{n=0, \ldots, N_{\varepsilon}} \mathbb{E}\left[\mathbb{E}\left[\left|\sup _{s \in\left[0, t_{1}\right]}\right| h\left(X_{s}^{\varepsilon}(y)\right)-h\left(\left.X_{s-t_{n}}(y)\right|^{p} \mid y=X_{t_{n}}^{\varepsilon}\left(x_{0}\right)\right]\right]^{\frac{1}{p}}\right. \\
& \leqslant T \varepsilon^{\lambda} \max _{n=0, \ldots, N_{\varepsilon}} \mathbb{E}\left[k_{4}\left(X_{t_{n}}^{\varepsilon}\left(x_{0}\right)\right)\right] .
\end{aligned}
$$


Note that by Corollary (3.2) we have

$$
\max _{n=0, \ldots, N_{\varepsilon}} \mathbb{E}\left[k_{4}\left(X_{t_{n}}^{\varepsilon}\left(x_{0}\right)\right)\right] \leqslant k_{3} k_{5}\left(1+\max _{n=0, \ldots, N_{\varepsilon}} \mathbb{E}\left[\bar{\eta}\left(X_{t_{n}}^{\varepsilon}\left(x_{0}\right)\right)\right]\right) .
$$

It remains to bound the last summand. We estimate as follows for any $n \in \mathbb{N}$

$$
\begin{aligned}
& \mathbb{E}\left[\bar{\eta}\left(X_{t_{n}}^{\varepsilon}\left(x_{0}\right)\right)\right] \\
& \leqslant \mathbb{E}\left[\bar{\eta}\left(X_{t_{n}}^{\varepsilon}\left(x_{0}\right)\right)-\bar{\eta}\left(X_{t_{n}}\left(x_{0}\right)\right)\right]+\mathbb{E}\left[\bar{\eta}\left(X_{t_{n}}\left(x_{0}\right)\right)\right] \\
& \leqslant \bar{\ell} \mathbb{E}\left[\left|X_{t_{n}}^{\varepsilon}\left(x_{0}\right)-X_{t_{n}}\left(x_{0}\right)\right|\right]+\int \bar{\eta}(y) \mu_{x_{0}}(d y)+\sup _{t \geqslant 0} \mathbb{E}\left[\left|\int \bar{\eta}(y) \mu_{x_{0}}(d y)-\bar{\eta}\left(X_{t}\left(x_{0}\right)\right)\right|\right] \\
& \leqslant \bar{\ell} \mathbb{E}\left[\left|X_{t_{n}}^{\varepsilon}\left(x_{0}\right)-X_{t_{n}}\left(x_{0}\right)\right|^{p}\right]^{\frac{1}{p}}+C_{1} .
\end{aligned}
$$

For the first term in the preceding expression we derive a recursion formula. Using Theorem 3.2 in Kunita [20] it yields for the horizontal component

$$
\mathbb{E}\left[\sup _{s \in 0, T]}\left|X_{s}\left(x_{1}\right)-X_{s}\left(x_{2}\right)\right|^{p}\right] \leqslant e^{\ell L i p\left(\varphi^{-1}\right) T}\left|x_{1}-x_{2}\right|^{p},
$$

which implies the inequality

$$
\mathbb{E}\left[\left|X_{t_{1}}\left(x_{1}\right)-X_{t_{1}}\left(x_{2}\right)\right|^{p}\right] \leqslant C_{2} \varepsilon^{\lambda}\left|x_{1}-x_{2}\right|^{p} .
$$

We estimate

$$
\begin{aligned}
& \mathbb{E}\left[\left|X_{t_{n}}^{\varepsilon}\left(x_{0}\right)-X_{t_{n}}\left(x_{0}\right)\right|^{p}\right]^{\frac{1}{p}} \\
& \left.\leqslant \mathbb{E}\left[\left|X_{t_{n}}^{\varepsilon}\left(x_{0}\right)-X_{t_{n}-t_{n-1}}\left(X_{t_{n-1}}^{\varepsilon}\left(x_{0}\right)\right)\right|^{p}\right]^{\frac{1}{p}}+\left.\mathbb{E}\left[\mid X_{t_{n}}\left(X_{t_{n-1}}^{\varepsilon}\left(x_{0}\right)\right)-X_{t_{n}}\left(x_{0}\right)\right)\right|^{p}\right]^{\frac{1}{p}} \\
& \leqslant C_{3} \varepsilon^{\lambda} \mathbb{E}\left[k_{4}\left(X_{t_{n-1}}^{\varepsilon}\left(x_{0}\right)\right)\right]+C_{2} \varepsilon^{\lambda} \mathbb{E}\left[\left|X_{t_{n-1}}^{\varepsilon}\left(x_{0}\right)-X_{t_{n-1}}\left(x_{0}\right)\right|^{p}\right]^{\frac{1}{p}} \\
& \leqslant C_{4} \varepsilon^{\lambda}\left(1+\mathbb{E}\left[\bar{\eta}\left(X_{t_{n-1}}^{\varepsilon}\left(x_{0}\right)\right)\right]\right)+C_{2} \varepsilon^{\lambda} \mathbb{E}\left[\left|X_{t_{n-1}}^{\varepsilon}\left(x_{0}\right)-X_{t_{n-1}}\left(x_{0}\right)\right|^{p}\right]^{\frac{1}{p}} \\
& \leqslant C_{4} \varepsilon^{\lambda}\left(C_{1}+\bar{\ell} \mathbb{E}\left[\left|X_{t_{n-1}}^{\varepsilon}\left(x_{0}\right)-X_{t_{n-1}}\left(x_{0}\right)\right|^{p}\right]^{\frac{1}{p}}\right)+C_{2} \varepsilon^{\lambda} \mathbb{E}\left[\left|X_{t_{n-1}}^{\varepsilon}\left(x_{0}\right)-X_{t_{n-1}}\left(x_{0}\right)\right|^{p}\right]^{\frac{1}{p}} \\
& =C_{5} \varepsilon^{\lambda} \mathbb{E}\left[\left|X_{t_{n-1}}^{\varepsilon}\left(x_{0}\right)-X_{t_{n-1}}\left(x_{0}\right)\right|^{p}\right]^{\frac{1}{p}}+C_{6} \varepsilon^{\lambda} .
\end{aligned}
$$

That is, for $\psi_{n}:=\mathbb{E}\left[\left|X_{t_{n}}^{\varepsilon}\left(x_{0}\right)-X_{t_{n}}\left(x_{0}\right)\right|^{p}\right]^{\frac{1}{p}}$ we have then

$$
\psi_{n} \leqslant C_{7} \varepsilon^{\lambda} \psi_{n-1}+C_{7} \varepsilon^{\lambda}
$$

which gives the following estimate for any $n \in \mathbb{N}$ and $k \in\{1, \ldots, n\}$

$$
\psi_{n} \leqslant\left(C_{7} \varepsilon^{\lambda}\right)^{n-k}+\sum_{i=1}^{n-k}\left(C_{7} \varepsilon^{\lambda}\right)^{i} .
$$

For $C_{7} \varepsilon_{0}^{\lambda}<\frac{1}{2}$ we obtain for any $n \in \mathbb{N}$ the estimate

$$
\psi_{n} \leqslant C_{7} \varepsilon^{\lambda}+\sum_{i=1}^{\infty}\left(C_{7} \varepsilon^{\lambda}\right) \leqslant 3 C_{7} \varepsilon^{\lambda}<\infty .
$$


Under these assumptions, we obtain for all $\varepsilon \in\left(0, \varepsilon_{0}\right]$

$$
\max _{n=0, \ldots, N_{\varepsilon}} \mathbb{E}\left[\bar{\eta}\left(X_{t_{n}}^{\varepsilon}\left(x_{0}\right)\right)\right] \leqslant \max _{n=0, \ldots, N_{\varepsilon}}\left(\bar{\ell} \mathbb{E}\left[\left|X_{t_{n}}^{\varepsilon}\left(x_{0}\right)-X_{t_{n}}\left(x_{0}\right)\right|^{p}\right]^{\frac{1}{p}}+C_{1}\right) \leqslant C_{7} \varepsilon^{\lambda}+C_{1}<\infty .
$$

Going back to our main estimate, we obtain $C_{8}>0$ such that

$$
\mathbb{E}\left[\left.\sup _{s \in[0, T]} A_{1}(s, \varepsilon)\right|^{p}\right]^{\frac{1}{p}} \leqslant T \varepsilon^{\lambda} k_{3} k_{5}\left(1+\max _{n=0, \ldots, N_{\varepsilon}} \mathbb{E}\left[\bar{\eta}\left(X_{t_{n}}^{\varepsilon}\left(x_{0}\right)\right)\right]\right) \leqslant C_{8} T \varepsilon^{\lambda}
$$

Lemma 4.3 For any $\lambda \in\left(0, \frac{p-1}{p}\right)$ there exist positive constants $b_{3}>0$ and $\varepsilon_{0} \in(0,1]$ such that for any $\varepsilon \in\left(0, \varepsilon_{0}\right]$ and $T \geqslant 0$

$$
\left(\mathbb{E}\left[\sup _{s \in[0, T]}\left|A_{2}(s, \varepsilon)\right|^{p}\right]\right)^{\frac{1}{p}} \leqslant b_{3} T \eta^{0}(c T|\ln (\varepsilon)|) .
$$

Proof: We have

$$
\begin{aligned}
\left(\mathbb{E}\left[\sup _{s \in[0, T]}\left|A_{2}(s, \varepsilon)\right|^{p}\right]\right)^{\frac{1}{p}} & \leqslant \varepsilon\left[\mathbb{E}\left|\sum_{n=0}^{N_{\varepsilon}-1}\left[\int_{t_{n}}^{t_{n+1}} h\left(X_{s-t_{n}}\left(X_{t_{n}}^{\varepsilon}\left(x_{0}\right)\right)\right) d s-\Delta_{\varepsilon} Q^{h}\left(\pi\left(X_{t_{n}}^{\varepsilon}\left(x_{0}\right)\right)\right)\right]\right|^{p}\right]^{\frac{1}{p}} \\
& \leqslant \varepsilon \Delta_{\varepsilon} \sum_{n=0}^{N_{\varepsilon}-1}\left[\mathbb{E}\left|\frac{1}{\Delta_{\varepsilon}} \int_{t_{n}}^{t_{n+1}} h\left(X_{s-t_{n}}\left(X_{t_{n}}^{\varepsilon}\left(x_{0}\right)\right)\right) d s-Q\left(\pi\left(X_{t_{n}}^{\varepsilon}\left(x_{0}\right)\right)\right)\right|^{p}\right]^{\frac{1}{p}} .
\end{aligned}
$$

We apply the Markov property for all $n=0, \ldots, N_{\varepsilon}$. By Hypothesis 3 the two terms inside the modulus converge to each other when $\Delta_{\varepsilon}$ goes to infinity with rate of convergence bounded by $\bar{\eta}\left(X_{t_{n}}^{\varepsilon}\left(x_{0}\right)\right) \eta^{0}\left(\Delta_{\varepsilon}\right)$. Hence, for small $\varepsilon$ we have

$$
\begin{aligned}
\left(\mathbb{E}\left[\sup _{s \in[0, T]}\left|A_{2}(s, \varepsilon)\right|^{p}\right]\right)^{\frac{1}{p}} & \leqslant \varepsilon N_{\varepsilon} \Delta_{\varepsilon} \eta^{0}\left(\Delta_{\varepsilon}\right) \max _{n=0, \ldots, N_{\varepsilon}} \mathbb{E}\left[\bar{\eta}\left(X_{t_{n}}^{\varepsilon}\left(x_{0}\right)\right)\right] \\
& \leqslant T \eta^{0}(c T|\ln (\varepsilon)|) \max _{n=0, \ldots, N_{\varepsilon}} \mathbb{E}\left[\bar{\eta}\left(X_{t_{n}}^{\varepsilon}\left(x_{0}\right)\right)\right] .
\end{aligned}
$$

Therefore, using (47), we obtain for $\varepsilon \in\left(0, \varepsilon_{0}\right]$ the estimate

$$
\left(\mathbb{E}\left[\sup _{s \in[0, T]}\left|A_{2}(s, \varepsilon)\right|^{p}\right]\right)^{\frac{1}{p}} \leqslant C_{8} T \eta^{0}(c T|\ln (\varepsilon)|) .
$$

Lemma 4.4 For any $\lambda \in\left(0, \frac{p-1}{p}\right)$ there exist positive constants $b_{4}>0$ and $\varepsilon_{0} \in(0,1]$ such that for any $\varepsilon \in\left(0, \varepsilon_{0}\right]$ and $T \geqslant 0$

$$
\left(\mathbb{E}\left[\sup _{s \in[0, T]}\left|A_{3}(s, \varepsilon)\right|^{p}\right]\right)^{\frac{1}{p}} \leqslant b_{4} T \varepsilon^{\lambda}
$$


Proof: We calculate

$$
\begin{aligned}
\left|A_{3}(T, \varepsilon)\right| & =\left|\sum_{n=0}^{N_{\varepsilon}} \varepsilon \Delta_{\varepsilon} Q^{\pi K}\left(\pi\left(X_{t_{n}}^{\varepsilon}\right)\right)-\int_{0}^{N_{\varepsilon} \Delta_{\varepsilon}} \varepsilon Q^{\pi K}\left(\pi\left(X_{\frac{s}{\varepsilon}}^{\varepsilon}\right)\right) d s\right| \\
& \leqslant \varepsilon \sum_{n=0}^{N_{\varepsilon}} \Delta_{\varepsilon} \sup _{t_{n} \leqslant s<t_{n+1}}\left|Q^{\pi K}\left(\pi\left(X_{s}^{\varepsilon}\right)\right)-Q^{\pi K}\left(\pi\left(X_{t_{n}}^{\varepsilon}\right)\right)\right| \\
& \leqslant \varepsilon \Delta_{\varepsilon} C_{1} \sum_{n=0}^{N_{\varepsilon}} \sup _{t_{n} \leqslant s<t_{n+1}}\left|v_{s}^{\varepsilon}-v_{t_{n}}^{\varepsilon}\right| .
\end{aligned}
$$

By Minkowski's inequality, the Markov property, Proposition 4.1 and (47) (with the appropriate constant $C_{8}$ ) we have hat

$$
\begin{aligned}
\mathbb{E}\left[\sup _{s \in[0, T]}\left|A_{3}(s, \varepsilon)\right|^{p}\right]^{\frac{1}{p}} & \leqslant T C_{1} \max _{n \in\left\{0, \ldots, N_{\varepsilon}\right\}} \mathbb{E}\left[\mathbb{E}\left[\sup _{t_{n} \leqslant s<t_{n+1}}\left|v_{s-t_{n}}^{\varepsilon}(y)-v_{0}^{\varepsilon}(y)\right|^{p} \mid y=\mathcal{F}_{t_{n}}\right]\right]^{\frac{1}{p}} \\
& \leqslant T C_{1} \max _{n \in\left\{0, \ldots, N_{\varepsilon}\right\}} \mathbb{E}\left[\mathbb{E}\left[\sup _{t_{0} \leqslant s<t_{1}}\left|v_{s}^{\varepsilon}(y)-v_{0}^{\varepsilon}(y)\right|^{p} \mid y=X_{t_{n}}^{\varepsilon}\left(x_{0}\right)\right]\right]^{\frac{1}{p}} \\
& \leqslant T C_{2} \varepsilon^{\lambda} \mathbb{E}\left[k_{4}\left(X_{t_{n}}^{\varepsilon}\left(x_{0}\right)\right)\right] \\
& \leqslant T C_{2} C_{8} \varepsilon^{\lambda} .
\end{aligned}
$$

This ends the proof of Proposition 4.1.

Proof of the main Theorem 2.4; $\quad$ With the help of Proposition 4.1, the proof of Theorem 2.4 is identical the one given in Section 5 of [7].

\section{Appendix: Nonlinear comparison principle}

Proposition 5.1 (Pachpatte [24]) Let $u, f, g$ and $h$ be nonnegative continuous functions defined on $\mathbb{R}^{+}$. Let $v$ be a continuous non-decreasing subadditive and submultiplicative function defined on $\mathbb{R}^{+}$and $v(u)>0$ on $(0, \infty)$. Let $e, \varphi$ be continuous and nondecreasing functions defined on $\mathbb{R}^{+}$ with $p$ being strictly positive and $\varphi(0)=0$. If

$$
u(t) \leqslant e(t)+g(t) \int_{0}^{t} f(s) u(s) d s+\varphi\left(\int_{0}^{t} h(s) v(u(s)) d s\right)
$$

for all $t \geqslant 0$, then for any $0 \leqslant t \leqslant t_{2}$

$$
u(t) \leqslant a(t)\left[e(t)+\varphi\left(F^{-1}\left(F(A(t))+\int_{0}^{t} h(s) v(a(s)) d s\right)\right)\right]
$$

where

$$
\begin{aligned}
& a(t):=1+g(t) \int_{0}^{t} f(s) \exp \left(\int_{s}^{t} g(\sigma) f(\sigma) d \sigma\right) d s \\
& A(t):=\int_{0}^{t} h(s) v(a(s) e(s)) d s
\end{aligned}
$$




$$
F(t):=\int_{0}^{t} \frac{d s}{v(\varphi(s))}
$$

$F^{-1}$ is the inverse of $F$ and $t_{2} \in \mathbb{R}^{+}$such that

$$
F(A(t))+\int_{0}^{t} h(s) v(a(t)) d s \in \operatorname{dom}\left(F^{-1}\right) \quad \text { for all } 0 \leqslant t \leqslant t_{2} .
$$

In the following special case of coefficients it is possible to drop the continuity assumption on $u$.

Corollary 5.2 Let $\Psi$ a non-negative, measurable, increasing function and $h$ be nonnegative, continuous, increasing function on the interval $[0, T]$ satisfying for $p \geqslant 2, \varepsilon>0, c>0$ and any $t \in[0, T]$ the inequality

$$
\Psi(t) \leqslant \varepsilon c t^{p}+\varepsilon c\left(\int_{0}^{t} \Psi(s)+\Psi(s)^{\frac{p-1}{p}} d s\right), \quad t \in[0, T] .
$$

Then there is a constant $k>0$ such that for any $\varepsilon_{0} \in(0,1]$ such that $\varepsilon_{0} T<k$ we have for all $t \in[0, T]$ and $\varepsilon \in\left(0, \varepsilon_{0}\right]$

$$
\Psi(t) \leqslant C\left(\varepsilon t^{p}+t^{p}(\varepsilon t)^{\frac{p-1}{p}}\right) .
$$

Proof: For $e(t)=c \varepsilon t^{p}, g \equiv 1, f, h \equiv \varepsilon c, \varphi(t)=t, w(t)=t^{\frac{p-1}{p}}$ we calculate the coefficients of Proposition 5.1

$$
a(t):=1+\varepsilon c \int_{0}^{t} \exp (\varepsilon c(t-s)) d s=\exp (\varepsilon c t)
$$

and in the limit of $\varepsilon t$ being small $(\varepsilon t \ll 1)$ we have

$$
\varepsilon \int_{0}^{t} a(s)^{\frac{p-1}{p}} d s=\varepsilon t\left(\frac{\exp \left(c \frac{p-1}{p} \varepsilon t\right)-1}{c \frac{p-1}{p} \varepsilon t}\right) \leqslant \varepsilon t \ll 12 \varepsilon t .
$$

Applying the change of parameter $r=\varepsilon s$ it follows that

$$
\begin{aligned}
A(t) & :=\int_{0}^{t} \exp \left(\varepsilon c \frac{p-1}{p} s\right)(e(s))^{\frac{p-1}{p}} d s=\int_{0}^{t} \exp \left(c \frac{p-1}{p} \varepsilon s\right)\left(c \varepsilon s^{p}\right)^{\frac{p-1}{p}} d s \\
& =\varepsilon^{\frac{p-1}{p}} \int_{0}^{\varepsilon t} \exp \left(c \frac{p-1}{p} r\right) c^{\frac{p-1}{p}}\left(\frac{r}{\varepsilon}\right)^{p-1} \frac{d r}{\varepsilon} \leqslant t \frac{1}{\varepsilon^{p} t} \int_{0}^{\varepsilon t} \exp \left(c \frac{p-1}{p} r\right)(c r)^{\frac{p-1}{p}} d r \\
& \leqslant \varepsilon t \ll 12 t \exp \left(c \frac{p-1}{p} \varepsilon t\right)(c \varepsilon t)^{\frac{p-1}{p}} \leqslant C_{1} t \exp \left(c \frac{p-1}{p} \varepsilon t\right)(\varepsilon t)^{\frac{p-1}{p}} .
\end{aligned}
$$

Finally, we obtain

$$
F(t):=\int_{0}^{t} s^{-\frac{p-1}{p}} d s=p r^{\frac{1}{p}} \quad \text { and } \quad F^{-1}(t):=\frac{t^{p}}{p^{p}} .
$$

In the sequel we follow the proof of Theorem 2.4.2 in Pachpatte 24] and define the continuous, positive, non-decreasing function

$$
n(t):=e(t)+\varphi\left(\int_{0}^{t} h(s) w(u(s)) d s\right)=e(t)+\varepsilon c \int_{0}^{t} h(s) u(s)^{\frac{p-1}{p}} d s, \quad t \geqslant 0,
$$


such that inequality (49) can be restated as

$$
u(t) \leqslant n(t)+g(t) \int_{0}^{t} f(s) u(s) d s=e(t)+\varepsilon c \int_{0}^{t} u(s) d s .
$$

It is well-known, see for instance [1, that this integral estimate implies the following GronwallBellmann inequality also in the case of $u$ being merely positive measurable. The main reason is that the integral is absolutely continuous with a bounded density. This result yields

$$
u(t) \leqslant a(t) n(t), \quad t \geqslant 0 .
$$

The remainder of the proof of Theorem 2.4.2 in [24] does use the continuity of $u$ and remains intact.

\section{Acknowledgements:}

The author PHC would like to thank the Department of Mathematics of Brasilia University for providing support. The authors MAH and PRR would like express his gratitude for the hospitality received at the Departameto de Matemática at Universidade de Brasília and the IMECC at UNICAMP in February 2018. The funding of MAH by the FAPA project "Stochastic dynamics of Lévy driven systems" at the School of Science at Universidad de los Andes is greatly acknowledged. The author PRR is partially supported by Brazilian CNPq proc. nr. 305462/2016-4, by FAPESP proc. nr. 2015/07278-0 and 2015/50122-0.

\section{References}

[1] Amann, H.: Ordinary differential equations. An introduction to nonlinear analysis. de Gruyter Studies in Mathematics, 13. Walter de Gruyter \& Co., 1990.

[2] Applebaum, D.: Lévy processes and stochastic calculus. Cambridge university press, 2nd edition, 2009.

[3] Arnold, V.: Mathematical Methods in Classical Mechanics. Springer, 2nd edition, 1989.

[4] Borodin, A., Freidlin, M.: Fast oscillating random perturbations of dynamical systems with conservation laws. Ann. Inst. H. Poincaré. Prob. Statist. 31, 485-525 (1995).

[5] Cannas, A.: Lectures on Symplectic Geometry. Lecture Notes in Mathematics 1764, 2008.

[6] Cerrai, S.: A Khasminskii type averaging principle for stochastic reaction-diffusion equations. Ann. Probab. 19(3), 899-948 (2009).

[7] da Costa, P.H., Högele, M.A.: Strong averaging along foliated Lévy diffusions with heavy tails on compact leaves. Potential Analysis 47(3), 277-311 (2017).

[8] Xu, Y., Duan, J., Xu, W.: An averaging principle for stochastic dynamical systems with Lvy noise, Physica D 240, 1395-1401 (2011).

[9] Freidlin, M.I., Wentzell, A.D.: Random Perturbations of Dynamical Systems. Springer-Verlag, 1991. 
[10] Gargate, I.I.G., Ruffino, P.R.: An averaging principle for diffusions in foliated spaces. Ann. Probab. 44 (1), 567-588 (2016).

[11] Garnett, L.: Foliation, the ergodic theorem and Brownian motion, Journal of Functional Analysis 51, 285-311 (1983).

[12] Högele, M.A., Ruffino, P.R.: Averaging along foliated Lévy diffusions. Nonlinear Analysis 112, 1-14 (2015).

[13] Kabanov, Y., Pergamenshchikov, S.: Two-Scale Stochastic Systems: asymptotic analysis and control. Springer-Verlag, 2003.

[14] Kakutani, S., Petersen, K.: The speed of convergence in the ergodic theorem. Monat. Mathematik 91, 11-18 (1981).

[15] Khasminski, R., Krylov, N.: On averaging principle for diffusion processes with null-recurrent fast component. Stoch Proc Appl. 93, 229-240 (2001).

[16] Bakhtin, V., Kifer, Y.: Nonconvergence examples in averaging. Geometric and probabilistic structures in dynamics. Contemp. Math. 469, 1-17 (2008).

[17] Krengel, U.: On the speed of convergence of the ergodic theorem. Monat. Mathematik 86, 3-6 (1978).

[18] Kurtz, T.G., Pardoux, E., Protter, Ph.: Stratonovich stochastic differential equations driven by general semimartingales. Annales de l'H.I.P., section B 31(2), 351-377 (1995).

[19] Kulik, A.: Exponential ergodicity of the solutions of SDE's with a jump noise. Stochastic Processes and their Applications 119, 602-632 (2009).

[20] Kunita, H.: Stochastic differential equations based on Lévy processes and stochastic flows of diffeomorphisms. In: Rao, M.M. (ed.) Real and Stochastic Analysis. Birkhäuser, 305-373 (2004).

[21] Li, X.-M.: An averaging principle for a completely integrable stochastic Hamiltonian systems. Nonlinearity 21, 803-822 (2008).

[22] Namachchvaya, S., Sowers, R.: Rigorous stochastic averaging at a center with additive noise. Meccanica 37, 85-114 (2002).

[23] Nash, J.: The imbedding problem for Riemannian manifolds. Annals of Mathematics 63 (1), 20-63 (1956).

[24] Pachpatte, B.G.: Inequalities for differential and integral equations. Academic Press , 1998.

[25] Protter, Ph.: Stochastic integration and differential equations. Springer-Verlag, 2004.

[26] Sato, K.-I.: Lévy processes and infinitely divisible distributions. Probab. Theory Relat. Fields 111, 287-321 (1998).

[27] Sowers, R.: Stochastic averaging with a flattened Hamiltonian: a Markov process on a stratified space (a whiskered sphere). Trans. Am. Math. Soc 354, 853-900 (2002).

[28] Sanders, J.A., Verhulst, F., Murdock, J.: Averaging Methods in Nonlinear dynamical Systems. Springer, 2nd edition, 2007. 
[29] Tondeur, P.: Foliations on Riemannian manifolds. Universitext, Springer-Verlag, 1988.

[30] Volsov, V.M.: Some types of calculation connected with averaging in the theory of non-linear vibrations. USSR Computational Mathematics and Mathematical Physics, 3(1), 1962.

[31] Volsov, V.M., Morgunov, B.I.: Methods of calculating stationary resonance vibrational and rotational motions of certain non-linear systems. USSR Computational Mathematics and Mathematical Physics 8(2), 1968.

[32] Walcak, P.: Dynamics of foliations, groups and pseudogroups. Birkhäuser Verlag, 2004. 